\title{
Guaranteed Conditional Performance of Control Charts via Bootstrap Methods
}

\author{
Axel Gandy \\ Departments of Mathematics, Imperial College London \\ Jan Terje Kvaløy \\ Department of Mathematics and Natural Sciences, \\ University of Stavanger, Norway
}

\begin{abstract}
To use control charts in practice, the in-control state usually has to be estimated. This estimation has a detrimental effect on the performance of control charts, which is often measured for example by the false alarm probability or the average run length. We suggest an adjustment of the monitoring schemes to overcome these problems. It guarantees, with a certain probability, a conditional performance given the estimated in-control state. The suggested method is based on bootstrapping the data used to estimate the in-control state. The method applies to different types of control charts, and also works with charts based on regression models, survival models, etc. If a nonparametric bootstrap is used, the method is robust to model errors. We show large sample properties of the adjustment. The usefulness of our approach is demonstrated through simulation studies.
\end{abstract}

Key words: Monitoring, CUSUM, bootstrap, guaranteed performance, confidence interval, control chart

\section{Introduction}

Control charts such as the Shewhart chart (Shewhart, 1931) and the cumulative sum (CUSUM) chart (Page, 1954) have been valuable tools in many areas, including reliability (O'Connor, 2002; Xie et al., 2002), medicine (Carey, 2003; Lawson and Ken, 2005; Woodall, 2006) and finance (Frisén, 2008). See Stoumbos et al. (2000) and the special issues of "Sequential Analysis" (2007, Volume 26 , Issues 2,3 ) for an overview. Often, heterogeneity between observations is accounted for by using risk-adjusted charts based on fitted regression models (Grigg and Farewell, 2004; Horváth et al. 2004 Gandy et al., 2010).

A common convention in monitoring based on control charts is to assume the probability distribution of in-control data to be known. In practice this usually means that the distribution is estimated based on a sample of in-control data and the estimation error is ignored. Examples of this are Steiner et al. (2000); Grigg and Farewell (2004); Bottle and Aylin (2008); Biswas and Kalbfleisch (2008); Fouladirad et al. (2008); Sego et al. (2009); Gandy et al. (2010).

However, the estimation error has a profound effect on the performance of control charts. This has been mentioned at several places in the literature, e.g. Jones et al. (2004); Albers and Kallenberg (2004b); Jensen et al. (2006); Stoumbos et al. (2000); Champ and Jones-Farmer (2007).

To illustrate the effect of estimation, we consider a CUSUM chart (Page, 1954) with normal observations and estimated in-control mean. We observe a stream of independent random variables $X_{1}, X_{2}, \ldots$ which in control have an $N(\mu, 1)$ distribution and out of control have an $N(\mu+\Delta, 1)$ distribution, where $\Delta>0$ is the shift in the mean. The chart switches from the in-control state to the out-of-control state at an unknown time $\kappa$. The unknown in-control mean $\mu$ is estimated by the average $\hat{\mu}$ of $n$ past in-control observations $X_{-n}, \ldots, X_{-1}$ (this is often called phase 1 of the 


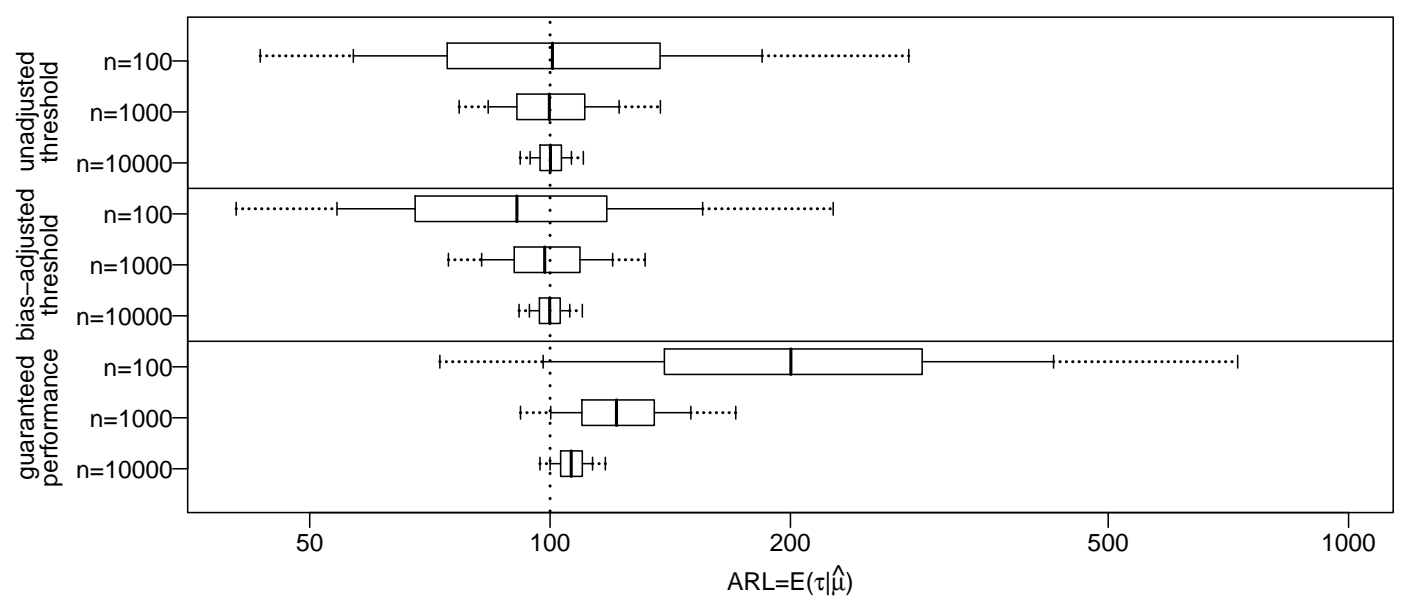

Figure 1: In-control distribution of $\mathrm{ARL}=\mathrm{E}(\tau \mid \hat{\mu})$ for CUSUMs for standard normally distributed data. The mean $\hat{\mu}$ used in the monitoring is estimated based on $n$ past observations. The boxplots show the $2.5 \%, 10 \%, 25 \%, 50 \%, 75 \%, 90 \%$ and $97.5 \%$ quantiles. The top part of the plot shows the situation when estimation error is ignored. In the middle part the threshold has been chosen to give an unconditional ARL of 100 (averaging out the parameter estimation). In the bottom part the threshold is adjusted to guarantee with $90 \%$ probability an in-control ARL of at least 100.

monitoring; the running of the chart is called phase 2). We consider the CUSUM chart

$$
S_{t}=\max \left(0, S_{t-1}+X_{t}-\hat{\mu}-\Delta / 2\right), \quad S_{0}=0
$$

with hitting time $\tau=\inf \left\{t>0: S_{t} \geq c\right\}$ for some threshold $c>0$.

The in-control average run length, $\mathrm{ARL}=\mathrm{E}(\tau \mid \hat{\mu}, \kappa=\infty)$, depends on $\hat{\mu}$ and is thus a random quantity. The top part of the plot in Figure 1 shows boxplots of its distributions with threshold $c=2.84, \Delta=1$ and various numbers of past observations. If $\hat{\mu}=\mu$, i.e. $\mu$ was know, this would give an in-control ARL of 100. The estimation error is having a substantial effect on the attained ARL even for large samples such as $n=1000$. For further illustrations of the impact of estimation error see Jones et al. (2004) for CUSUM charts and Albers and Kallenberg (2004b) for Shewhart charts.

So far, no general approach for taking the estimation error into account has been developed, but there are many special constructions for specific situations. For instance, for some charts so called self-starting charts (Hawkins, 1987; Hawkins and Olwell, 1998, Sullivan and Jones, 2002), maximum likelihood surveillance statistics to eliminate parameters (e.g. Frisén and Andersson, 2009), correction factors for thresholds (Albers and Kallenberg, 2004b; Jones, 2002), modified thresholds (Zhang et al., 2011) and threshold functions (Horváth et al., 2004 Aue et al., 2006) have been developed. Various bootstrap schemes for specific situations have also been suggested, see for instance Kirch (2008); Chatterjee and Qiu (2009); Capizzi and Masarotto (2009); Hušková and Kirch (2010). Further, some nonparametric charts which account for the estimation error in past data have been proposed, see Chakraborti and Graham (2007) and references therein. Recently some modified charts for monitoring variance in the normal distribution with estimated parameters have been suggested by Maravelakis and Castagliola (2009) and Castagliola and Maravelakis (2011).

When addressing estimation error, the above methods mainly focus on the performance of the charts averaged over both the estimation of the in-control state as well as running the chart once. In the middle part of Figure 1, the threshold has been chosen such that, averaged over both the estimation of the in-control state as well as running the chart once, the average run length is 100 (this results in a different threshold for each $n$ ). It turns out that only a small change in the threshold is needed and that the distribution of the conditional ARL $=\mathrm{E}(\tau \mid \hat{\mu})$ is only changed slightly. This bias correction for the ARL actually goes in the wrong direction in the sense that it implies more short ARLs. This is due to the ARL being substantially influenced by the right tail of the run length distribution, see the discussion in Section 2 of Albers and Kallenberg (2006). 
However, usually, after the chart parameters are estimated, the chart is run for some time without any reestimation of the in-control state even if the chart signals. Moreover, in some situations, several charts are run based on the same estimated parameters. In these situations the ARL conditional on the estimated in-control state is more relevant than the unconditional ARL. In the middle and upper part of Figure 1, one sees that the conditional ARL can be much lower than 100, meaning that both the unadjusted threshold and the threshold adjusted for bias in the unconditional ARL lead, with a substantial probability, to charts that have a considerably decreased time until false alarms.

To overcome these problems we will look at the performance of the chart conditional on the estimated in-control distribution, averaging only over different runs of the chart. This will lead to the construction of charts that with high probability have an in-control distribution with desired properties conditional on the observed past data, thus reducing the situations in which there are many false alarms due to estimation error.

The bottom part of Figure 1 shows the distribution of the in control ARL when the threshold for each set of past data is adjusted to guarantee an in-control ARL of at least 100 with probability $90 \%$. The adjustment is calculated using a bootstrap procedure explained later in the paper. The adjustment succeeds to avoid the too low ARLs with the prescribed probability, and we will see later that the cost in a higher out-of-control ARL is modest. Using hitting probabilities instead of ARL as criterion leads to similar results.

Our approach is similar in spirit to the exceedance probability concept developed by Albers and Kallenberg for various types of Shewhart (Albers and Kallenberg, 2004a, 2005, Albers et al., 2005) and negative binomial charts (Albers and Kallenberg, 2009, 2010). They calculate approximate adjusted thresholds such that there is only a small prescribed probability that some performance measure, for instance an ARL, will be a certain amount below or above a specified target.

The main difference between their approach and what we present is that our approach applies far more widely, to many different types of charts and without having to derive specific approximation formulas in each setting. If we apply a nonparametric bootstrap, the proposed procedure will be robust against model misspecification. In addition to that, our approach allows not only to adjust the threshold but also to give a confidence interval for the in-control performance of a chart for a fixed threshold. Lastly, even though not strongly advocated in this paper, the bootstrap procedure we propose can also be used to do a bias correction for the unconditional performance of the chart, as in the middle part of Figure 1 .

Next, we describe our approach more formally. Suppose we want to use a monitoring scheme and that the in-control distribution $P$ of the observations is unknown, but that based on past in-control behaviour we have an estimate $\hat{P}$ of the in-control distribution. Let $q$ denote the incontrol property of the chart we want to compute, such as the ARL, the false alarm probability or the threshold needed for a certain ARL or false alarm probability. In the above example we were interested to find a threshold such that the in-control ARL is 100 .

Generally, $q$ may depend on both the true in-control distribution $P$ and on estimated parameters of this distribution which for many charts are needed to run the chart. We denote these parameters by $\hat{\xi}=\xi(\hat{P})$. In the above CUSUM chart example $\hat{\xi}=\hat{\mu}$. We are interested in $q(P ; \hat{\xi})$, that is the in-control performance of the chart conditional on the estimated parameter. In the above CUSUM example, $q(P ; \hat{\xi})$ is the threshold needed to give an ARL of 100 if the observations are from the true in-control distribution $P$ and the estimated parameter $\hat{\mu}$ is used. As $P$ is not observed $q(P ; \hat{\xi})$ is not observable. As mentioned above, many papers pretend that the estimated in-control distribution $\hat{P}$ equals the true in-control distribution $P$ and thus use $q(\hat{P} ; \hat{\xi})$. Our suggestion is to use bootstrapping of past data to construct an approximate one-sided confidence intervals for $q(P ; \hat{\xi})$. From this we get a guaranteed conditional performance of the control scheme.

In Section 2 we present the general idea in the setting with homogeneous observations, and discuss this for Shewhart and CUSUM charts. The main theoretical results are presented in Section 3, with most of the proofs given in the Appendix. Section 4 contains simulations illustrating the performance of charts for homogeneous observations. In Section 5 extensions to charts based on regression and survival analysis models are presented. Some concluding comments are given in Section 6. The suggested methods are implemented in a flexible R-package, that will be made 
available on the Comprehensive R Archive Network (CRAN).

\section{Monitoring homogeneous observations}

\subsection{General idea}

Suppose that in control we have independent observations $X_{1}, X_{2}, \ldots$ following an unknown distribution $P$. We want to use some monitoring scheme/control chart that detects when $X_{i}$ is no longer coming from $P$. The particular examples we discuss in this paper are Shewhart and CUSUM charts, but the methodology we suggest applies more widely.

To run the charts, one often needs certain parameters $\xi$. For example, in the CUSUM control chart of the introduction, we needed $\xi=\mu$, the assumed in-control mean. These parameters will usually be estimated.

Let $\tau$ denote the time at which the chart signals a change. As $\tau$ may depend on $\xi$, we sometimes write $\tau(\xi)$. The charts we consider use a threshold $c$, which determines how quickly the chart signals (larger $c$ lead to a later signal).

The performance of such a control chart with the in-control distribution $P$ and the parameters $\xi$ can, for example, be expressed as one of the following.

- $\operatorname{ARL}(P ; \xi)=\mathrm{E}(\tau(\xi))$, where $\mathrm{E}$ is the expectation with respect to $P$.

- $\operatorname{hit}(P ; \xi)=\mathrm{P}(\tau(\xi) \leq T)$ for some finite $T>0$, where $\mathrm{P}$ is the probability measure under which $X_{1}, X_{2}, \cdots \sim P$. This is the false alarm probability in $T$ time units.

- $c_{\mathrm{ARL}}(P ; \xi)=\inf \{c>0: \operatorname{ARL}(P ; \xi) \geq \gamma\}$ for some $\gamma>0$. Assuming appropriate continuity, this is the threshold needed to give an in-control average run length of $\gamma$.

- $c_{\text {hit }}(P ; \xi)=\inf \{c>0: \operatorname{hit}(P ; \xi) \leq \beta\}$ for some $0<\beta<1$. This is the threshold needed to give a false alarm probability of $\beta$.

The latter two quantities are very important in practice, as they are needed to decide which threshold to use to run a chart. In the notation we have suppressed the dependence of the quantities on $c, T, \gamma, \beta$ and $\Delta$.

In the following, $q$ will denote one of ARL, hit, $c_{\mathrm{ARL}}$ or $c_{\text {hit }}$, or simple transformations such as $\log (\mathrm{ARL}), \operatorname{logit}(\mathrm{hit}), \log \left(c_{\mathrm{ARL}}\right)$ and $\log \left(c_{\mathrm{hit}}\right)$, where $\operatorname{logit}(x)=\log \left(\frac{x}{1-x}\right)$.

The true in-control distribution $P$ and the parameters $\xi=\xi(P)$ needed to run the chart are usually estimated. We assume that we have past in-control observations $X_{-n}, \ldots, X_{-1}$ (independent of $X_{1}, X_{2}, \ldots$ ), which we use to estimate the in-control distribution $P$ parametrically or non-parametrically. We denote this estimate by $\hat{P}$. The estimate of $\xi$ will be denoted by $\hat{\xi}=\xi(\hat{P})$. For example, in the CUSUM control chart of the introduction, $\hat{\xi}=\hat{\mu}$ is the estimated in-control mean.

The observed performance of the chart will depend on the true in-control distribution $P$ as well as on the estimated parameters $\hat{\xi}$ that are used to run the chart. Thus we are interested in $q(P ; \hat{\xi})$, the performance of the control chart conditional on $\hat{\xi}$. This is an unknown quantity as $P$ is not known. Based on the estimator $q(\hat{P} ; \hat{\xi})$, we construct a one-sided confidence interval for this quantity to guarantee, with high probability, a certain performance for the chart. We choose to call the interval a confidence interval, even though the quantity $q(P ; \hat{\xi})$ is random.

We suggest the following for guaranteeing an upper bound on $q$ (which is relevant for $q=$ hit, $q=c_{\mathrm{ARL}}$ or $\left.q=c_{\text {hit }}\right)$. For $\alpha \in(0,1)$, let $p_{\alpha}$ be a constant such that

$$
\mathrm{P}\left(q(\hat{P} ; \hat{\xi})-q(P ; \hat{\xi})>p_{\alpha}\right)=1-\alpha,
$$

assuming that such a $p_{\alpha}$ exists. Hence,

$$
\mathrm{P}\left(q(P ; \hat{\xi})<q(\hat{P} ; \hat{\xi})-p_{\alpha}\right)=1-\alpha .
$$

Thus $\left(-\infty, q(\hat{P} ; \hat{\xi})-p_{\alpha}\right)$ could be considered an exact lower one-sided confidence interval of $q(P ; \hat{\xi})$. 
Of course, $p_{\alpha}$ is unknown. We suggest to obtain an approximation of $p_{\alpha}$ via bootstrapping. In the following, $\hat{P}^{*}$ denotes a parametric or non-parametric bootstrap replicate of the estimated in-control distribution $\hat{P}$. We can approximate $p_{\alpha}$ by $p_{\alpha}^{*}$ such that

$$
\mathrm{P}\left(q\left(\hat{P}^{*} ; \hat{\xi}^{*}\right)-q\left(\hat{P} ; \hat{\xi}^{*}\right)>p_{\alpha}^{*} \mid \hat{P}\right)=1-\alpha .
$$

Thus

$$
\left(-\infty, q(\hat{P} ; \hat{\xi})-p_{\alpha}^{*}\right)
$$

is a one-sided (approximate) confidence interval for $q(P ; \hat{\xi})$. In this paper, we will use the following generic algorithm to implement the bootstrap.

Algorithm 1 (Bootstrap).

1. From the past data $\dot{X}_{-n}, \ldots, X_{-1}$, estimate $\hat{P}$ and $\hat{\xi}$.

2. Generate bootstrap samples $X_{-n}^{*}, \ldots, X_{-1}^{*}$ from $\hat{P}$. Compute the corresponding estimate $\hat{P}^{*}$ and $\hat{\xi}^{*}$. Repeat $B$ times to get $\hat{P}_{1}^{*}, \ldots, \hat{P}_{B}^{*}$ and $\hat{\xi}_{1}^{*}, \ldots, \hat{\xi}_{B}^{*}$.

3. Let $p_{\alpha}^{*}$ be the $1-\alpha$ empirical quantile of $q\left(\hat{P}_{b}^{*} ; \hat{\xi}_{b}^{*}\right)-q\left(\hat{P} ; \hat{\xi}_{b}^{*}\right), b=1, \ldots, B$.

For guaranteeing a lower bound on $q$, which is for example relevant for $q=$ ARL, a similar upper one-sided confidence interval can be constructed.

In a practical situation, the focus would be on deciding which threshold to use for the control chart to obtain desired in-control properties. We suggest to use either $q=c_{\mathrm{ARL}}$ or $q=c_{\text {hit }}$, or $\log$ transforms of these, and then run the chart with the adjusted threshold

$$
q(\hat{P} ; \hat{\xi})-p_{\alpha}^{*}
$$

This will guarantee that in (approximately) $1-\alpha$ of the applications of this method, the control chart actually has the desired in-control properties.

\subsection{Specific charts}

\subsubsection{Shewhart charts}

The one-sided Shewhart chart (Shewhart, 1931) signals at

$$
\tau=\inf \left\{t \in\{1,2, \ldots\}: f\left(X_{t}, \xi\right)>c\right\}
$$

for some threshold $c$, where $f$ is some function, $X_{t}$ is the observation at time $t$ and $\xi$ are some parameters. $X_{t}$ can be a single measurement or e.g. the average, range or standard deviation of a specified number of measurements, or some other statistic like a proportion. It is common to use a Shewhart chart with a threshold of the mean plus 3 times the standard deviation, in this case one would use $c=3$ and $f(x, \xi)=\frac{x-\xi_{1}}{\xi_{2}}$ with $\xi_{1}$ being the mean and $\xi_{2}$ being the standard deviation. For two-sided charts one could just use $f(x, \xi)=\frac{\left|x-\xi_{1}\right|}{\xi_{2}}$.

Conditionally on fixed parameters $\xi$, the stopping time $\tau$ follows a geometric distribution with parameter $p=p(c ; P, \xi)=\mathrm{P}\left(f\left(X_{t}, \xi\right)>c\right)$. Then the performance measures mentioned in the previous section simplify to

$$
\begin{aligned}
& \operatorname{ARL}(P ; \xi)=\frac{1}{p(c ; P, \xi)}, \\
& c_{\mathrm{ARL}}(P ; \xi)=p^{-1}\left(\frac{1}{\gamma} ; P, \xi\right) \quad \text { and } \quad c_{\mathrm{hit}}(P ; \xi)=p^{-1}\left(1-(1-\beta)^{\frac{1}{T}} ; P, \xi\right) \text {, }
\end{aligned}
$$

where $p^{-1}(\cdot ; P, \xi)$ is the inverse of $p(\cdot ; P, \xi)$.

Suppose that the in-control distribution comes from a parametric family $P_{\theta}, \theta \in \Theta$. Furthermore, suppose that we have some way of computing an estimate $\hat{\theta}$ of $\theta$ based on the sample. Then we can use Algorithm 1 with $\hat{P}=P_{\hat{\theta}}$ to compute a confidence interval as given by 1 .

Shewhart charts depend heavily on the tail behaviour of the distribution of the observations. This is particularly problematic when the sample size is small and we use non-parametric methods or a simple non-parametric bootstrap. We thus primarily suggest to use a parametric bootstrap for Shewhart charts. 
Remark 1. In certain cases the parametric bootstrap will actually be exact when $B \rightarrow \infty$. This happens when the distribution of $q\left(P_{\hat{\theta}} ; \hat{\xi}\right)-q\left(P_{\theta} ; \hat{\xi}\right)$ under $P_{\theta}$ does not depend on $\theta$. In particular, this implies that $q\left(P_{\hat{\theta}^{*}} ; \hat{\xi}^{*}\right)-q\left(P_{\hat{\theta}} ; \hat{\xi}^{*}\right)$ has the same distribution and $p_{\alpha}^{*} \rightarrow p_{\alpha}$ as $B \rightarrow \infty$.

As an example, consider the case when $f(x, \xi)=\frac{x-\xi_{1}}{\xi_{2}}$ and $X_{t}$ follows an $N\left(\xi_{1}, \xi_{2}^{2}\right)$ distribution and $q$ is any of the performance measures described above. We use $\theta=\xi$ and as estimator $\hat{\xi}_{1}$ we use the sample mean and as estimator $\hat{\xi}_{2}$ we use the sample standard deviation. Then

$$
p\left(c ; P_{\xi}, \hat{\xi}\right)=\mathrm{P}_{\xi}\left(\frac{X_{t}-\hat{\xi}_{1}}{\hat{\xi}_{2}}>c\right)=1-\Phi\left(\frac{c \hat{\xi}_{2}+\hat{\xi}_{1}-\xi_{1}}{\xi_{2}}\right),
$$

where $\Phi$ is the cdf of the standard normal distribution, and under $P_{\xi}$,

$$
\frac{c \hat{\xi}_{2}+\hat{\xi}_{1}-\xi_{1}}{\xi_{2}}=c \frac{\hat{\xi}_{2}}{\xi_{2}}+\frac{\hat{\xi}_{1}-\xi_{1}}{\xi_{2}} \sim \frac{c}{\sqrt{n-1}} \sqrt{W}+\frac{1}{\sqrt{n}} Z,
$$

where $W \sim \chi_{n-1}^{2}$ and $Z \sim N(0,1)$ are independent. Thus the distribution of $p\left(c ; P_{\xi}, \hat{\xi}\right)$, and hence $q\left(P_{\xi} ; \hat{\xi}\right)$, is completely known. As $p\left(c ; P_{\hat{\xi}}, \hat{\xi}\right)=\mathrm{P}_{\hat{\xi}}\left(\frac{X_{t}-\hat{\xi}_{1}}{\hat{\xi}_{2}}>c\right)=1-\Phi(c)$, and thus $q\left(P_{\hat{\xi}} ; \hat{\xi}\right)$, is not random, the distribution of $q\left(P_{\hat{\xi}} ; \hat{\xi}\right)-q\left(P_{\xi} ; \hat{\xi}\right)$ also does not depend on any unknown parameters. Thus the parametric bootstrap is exact in this example.

\subsubsection{CUSUM charts}

This section considers the one-sided CUSUM chart (Page, 1954). The classical CUSUM chart was designed to detect a shift of size $\Delta>0$ in the mean of normally distributed observations. Let $\mu$ and $\sigma$ denote, respectively, the in-control mean and standard deviation. A CUSUM chart can be defined by

$$
S_{t}=\max \left(0, S_{t-1}+\left(X_{t}-\mu-\Delta / 2\right) / \sigma\right), \quad S_{0}=0
$$

with hitting time $\tau=\inf \left\{t>0: S_{t} \geq c\right\}$ for some threshold $c>0$.

Alternatively, we could drop the scaling and not divide by the standard deviation $\sigma$ in (3). See Chapter 1.4 in Hawkins and Olwell (1998) for a discussion on scaled versus unscaled CUSUMs.

More generally, to accommodate observations with general in-control distribution with density $f_{0}$ and general out-of-control distribution with density $f_{1}$, it is optimal in a certain sense (Moustakides, 1986) to modify the CUSUM chart by replacing $\left(X_{t}-\mu-\Delta / 2\right) / \sigma$ by the log likelihood ratio $\log \left(f_{1}\left(X_{t}, \theta\right) / f_{0}\left(X_{t}, \theta\right)\right)$ such that the CUSUM chart is

$$
S_{t}=\max \left(0, S_{t-1}+\log \left(f_{1}\left(X_{t}, \theta\right) / f_{0}\left(X_{t}, \theta\right)\right)\right), \quad S_{0}=0 .
$$

Let $\xi$ denote either $(\mu, \sigma)$ in (3) or $\theta$ in (4). Usually, $\xi$ needs to be estimated from past data, and we can then use Algorithm 1 to compute a confidence interval (1) for the performance measure $q(P ; \hat{\xi})$. For 4 it is most natural to use a parametric bootstrap with $\hat{P}=P_{\hat{\theta}}$, while for 3 we can use either a parametric or a nonparametric bootstrap. In the latter case we let $\hat{P}$ be the empirical distribution of $X_{-n}, \ldots, X_{-1}$, i.e. in Algorithm 1, $X_{-n}^{*}, \ldots, X_{-1}^{*}$ are sampled with replacement from $X_{-n}, \ldots, X_{-1}$.

Remark 2. Similar as for Shewhart charts, this parametric bootstrap is exact when the distribution of $q\left(P_{\hat{\theta}} ; \hat{\xi}\right)-q\left(P_{\theta} ; \hat{\xi}\right)$ does not have any unknown parameters. This is, for instance, the case if we use (4) for an exponential distribution with the out-of-control distribution specified as an exponential distribution with mean $\Delta \lambda$, where $\lambda$ is the in-control mean. Another example of this is when we have normally distributed data and use a CUSUM with the increments $\left(X_{t}-\hat{\mu}\right) / \hat{\sigma}-\Delta / 2$.

\section{General theory}

In this section, we show that asymptotically, as the number of past observations $n$ increases, our procedure works. An established way of showing asymptotic properties of bootstrap procedures is 
via a functional delta method (van der Vaart and Wellner, 1996; Kosorok, 2008). Whilst we will follow a similar route, our problem does not fit directly into the standard framework, because the quantity of interest, $q(P, \hat{\xi})$, contains the random variable $\hat{\xi}$. We present the setup and the main result in Section 3.1, followed by examples (Section 3.2).

\subsection{Main theorem}

Let $D_{q}$ be the set in which $P$ and its estimator $\hat{P}$ lie, i.e. a set describing the potential probability distribution of our observations. This could be a subset of $\mathbb{R}^{d}$ for parametric distributions, the set of cumulative distribution functions for non-parametric situations, or the set of joint distributions of covariates and observations. We assume that $D_{q}$ is a subset of a complete normed vector space $D$. Let $\Xi$ be a non-empty topological space containing the potential parameters $\xi$ used for running the chart. In our examples, we will let $\Xi \subset \mathbb{R}^{d}$ be an open set.

We assume that $\hat{P}^{*}=\hat{P}^{*}\left(\hat{P}, W_{n}\right)$ is a bootstrapped version of $\hat{P}$ based both on the observed data $\hat{P}$ and on an independent random vector $W_{n}$. For example, when resampling with replacement then $W_{n}$ is a weight vector of length $n$, multinomially distributed, that determines how often a given observation is resampled. In a parametric bootstrap, $W_{n}$ is the vector of random variables needed to generate observations from the estimated parametric distribution.

In the main theorem we will need that the mapping $q: D_{q} \times \Xi \rightarrow \mathbb{R}$, which returns the property of the chart we are interested in, satisfies the following extension of Hadamard differentiability. For the usual definition of Hadamard differentiability see e.g. (van der Vaart, 1998, Section 20.2). The extension essentially consists in requiring Hadamard differentiability in the first component when the second component is converging.

Definition 1. Let $D, E$ be metric spaces, let $D_{f} \subset D$ and let $\Xi$ be a non-empty topological space. The family of functions $\left\{f(\cdot ; \xi): D_{f} \rightarrow E: \xi \in \Xi\right\}$ is called Hadamard differentiable at $\theta \in D_{f}$ around $\xi \in \Xi$ tangentially to $D_{0} \subset D$ if there exists a continuous linear map $f^{\prime}(\theta ; \xi): D_{0} \rightarrow E$ such that

$$
\frac{f\left(\theta+t_{n} h_{n} ; \xi_{n}\right)-f\left(\theta ; \xi_{n}\right)}{t_{n}} \rightarrow f^{\prime}(\theta ; \xi)(h) \quad(n \rightarrow \infty)
$$

for all sequences $\left(\xi_{n}\right) \subset \Xi,\left(t_{n}\right) \subset \mathbb{R},\left(h_{n}\right) \subset D$ that satisfy $\theta+t_{n} h_{n} \in D_{f} \forall n$ and $\xi_{n} \rightarrow \xi, t_{n} \rightarrow 0$, $h_{n} \rightarrow h \in D_{0}$ as $n \rightarrow \infty$.

In the following theorem we understand convergence in distribution, denoted by $\rightsquigarrow$, as defined in van der Vaart and Wellner (1996, Def 1.3.3) or in Kosorok (2008, p.108).

Theorem 1. Let $q: D_{q} \times \Xi \rightarrow \mathbb{R}$ be a mapping, let $P \in D_{q}$ and let $\xi: D_{q} \rightarrow \Xi$ be a continuous function. Suppose that the following conditions are satisfied.

a) $q$ is Hadamard differentiable at $P$ around $\xi$ tangentially to $D_{0}$ for some $D_{0} \subset D$.

b) $\hat{P}$ is a sequence of random elements in $D_{q}$ such that $\sqrt{n}(\hat{P}-P) \rightsquigarrow Z$ as $n \rightarrow \infty$ where $Z$ is some tight random element in $D_{0}$.

c) $\sqrt{n}\left(\hat{P}^{*}-\hat{P}\right) \underset{W}{\stackrel{P}{\rightarrow} Z}$ as $n \rightarrow \infty$ where $\underset{W}{\stackrel{P}{\sim}}$ denotes weak convergence conditionally on $\hat{P}$ in probability as defined in Kosorok (2008, p.19).

d) The cumulative distribution function of $q^{\prime}(P ; \xi) Z$ is continuous.

e) Outer-almost surely, the map $W_{n} \mapsto h\left(\hat{P}^{*}\left(\hat{P}, W_{n}\right)\right)$ is measurable for each $n$ and for every continuous bounded function $h: D_{q} \rightarrow \mathbb{R}$.

f) $q(\hat{P} ; \hat{\xi})-q(P ; \hat{\xi})$ and $p_{\alpha}^{*}$ are random variables, i.e. measurable, where $\hat{\xi}=\xi(\hat{P})$ and $p_{\alpha}^{*}=$ $\inf \left\{t \in \mathbb{R}: \hat{\mathrm{P}}\left(q\left(\hat{P}^{*} ; \hat{\xi}^{*}\right)-q\left(\hat{P} ; \hat{\xi}^{*}\right) \leq t\right) \geq \alpha\right\}$.

Then

$$
\mathrm{P}\left(q(P ; \hat{\xi}) \in\left(-\infty, q(\hat{P} ; \hat{\xi})-p_{\alpha}^{*}\right)\right) \rightarrow 1-\alpha \quad(n \rightarrow \infty) .
$$

A similar result holds for upper confidence intervals.

The proof is in Appendix A. The theorem essentially is an extension of the delta-method. Condition a) ensures the necessary differentiability. Conditions b) and c) are standard assumptions 
for the functional delta method; b) for the ordinary delta method and c) for the bootstrap version of it. Condition d) ensures that, after using an extension of the delta-method, the resulting confidence interval will have the correct asymptotic coverage probability. Condition e) is a technical measurability condition, which will be satisfied in our examples. Condition f) is a measurability condition, which should usually be satisfied.

\subsection{Examples}

The following sections give examples in which Theorem 1 applies. We consider hitting probabilities $(q=$ hit $)$ and thresholds to obtain certain hitting probabilities $\left(q=c_{\text {hit }}\right)$.

These examples are meant to be illustrative rather than exhaustive. For example, other parametric setups could be considered along similar lines to Section 3.2.2. Furthermore, other performance measures such as $\log \left(c_{\text {hit }}\right)$ or logit(hit) would essentially require application of chain rules to show differentiability.

\subsubsection{Simple nonparametric setup for CUSUM charts}

We show how the above theorem applies to the CUSUM chart described in (3) when using a non-parametric bootstrap version of Algorithm 1 .

Let $D=l_{\infty}(\mathbb{R})$ be the set of bounded functions $\mathbb{R} \rightarrow \mathbb{R}$ equipped with the sup-norm $\|x\|=$ $\sup _{t \in \mathbb{R}}\left|x_{t}\right|$. Let $D_{q} \subset D$ be the set of cumulative distribution functions on $\mathbb{R}$ with finite second moment. The parameters needed to run the chart are the mean and the standard deviation of the in-control observations, thus we may choose $\Xi=\mathbb{R} \times(0, \infty)$ and $\xi: D_{q} \rightarrow \Xi, P \mapsto$ $\left(\int x P(d x), \int x^{2} P(d x)-\left(\int x P(d x)\right)^{2}\right)$.

As quantities $q$ of interest we are considering hitting probabilities $(q=$ hit $)$ and thresholds $\left(q=c_{\text {hit }}\right)$ needed to achieve a certain hitting probability. The probability hit $: D_{q} \times \Xi \rightarrow \mathbb{R}$ of hitting a threshold $c>0$ up to step $T>0$ can be written as hit $(P ; \xi)=\mathrm{P}(m(Y) \geq c)$, where $m(Y)=\max _{i=1, \ldots, T} R_{i}(Y)$ is the maximum value of the chart up to time $T, R_{i}(Y)=\sum_{j=1}^{i} Y_{j}-$ $\min _{0 \leq k \leq i} \sum_{j=1}^{k} Y_{j}$ is the value of the CUSUM chart at time $i, Y=\left(Y_{1}, \ldots, Y_{T}\right), Y_{t}=\frac{X_{t}-\xi_{1}-\Delta / 2}{\xi_{2}}$ and $X_{1}, \ldots, X_{T} \sim P$ are the independent observations. The threshold needed to achieve a certain hitting probability $\beta \in(0,1)$ is $c_{\text {hit }}: D_{q} \times \Xi \rightarrow \mathbb{R}, c_{\text {hit }}(P ; \xi)=\inf \{c>0: \operatorname{hit}(P ; \xi) \leq \beta\}$.

The setup for the nonparametric bootstrap is as follows. $W_{n}$ is an $n$-variate multinomially distributed random vector with probabilities $1 / n$ and $n$ trials. The resampled distribution is $\hat{P}^{*}=\frac{1}{n} \sum_{j=1}^{n} W_{n j} \delta_{X_{-j}}$, where $\delta_{x}$ denotes the Dirac measure at $x$.

The following lemma shows condition a) of Theorem 1. the Hadamard differentiability of hit and $c_{\text {hit }}$.

Lemma 1. For every $P \in D_{q}$, and every $\xi \in \mathbb{R} \times(0, \infty)$, the function hit is Hadamard differentiable at $P$ around $\xi$ tangentially to $D_{0}=\left\{H: \mathbb{R} \rightarrow \mathbb{R}: H\right.$ continuous, $\lim _{t \rightarrow \infty} H(t)=\lim _{t \rightarrow-\infty} H(t)=$ $0\}$. If, in addition, $P$ has a continuous bounded positive derivative $f$ with $f(x) \rightarrow 0$ as $x \rightarrow \pm \infty$, then $c_{\text {hit }}$ is also Hadamard differentiable at $P$ around $\xi$ tangentially to $D_{0}$.

The proof is in Appendix B.4, with preparatory results in Appendix B.1-B.3

Conditions b) and c) of Theorem 1 follow directly from empirical process theory, see e.g. (Kosorok, 2008, p.17, Theorems 2.6 and 2.7). Condition e) is satisfied as well, see bottom of p.189 and after Theorem 10.4 (p.184) of Kosorok (2008).

Verifying condition d) in full is outside the scope of the present paper. A starting point could be the fact that by the Donsker theorem, $Z \sim G \circ P$, where $G$ is a Brownian bridge.

\subsubsection{CUSUM charts with normally distributed observations}

In this section, we consider a similar setup to the monitoring based on (3) considered in the previous subsection with the difference that we now use parametric assumptions. More specifically, the observations $X_{i}$ follow a normal distribution with unknown mean $\mu$ and variance $\sigma^{2}$. We will use this both for computing the properties of the chart as well as in the bootstrap, which will be a parametric bootstrap version of Algorithm 1 . 
The distribution of the observations can be identified with its parameters which we estimate by $\hat{P}=\left(\hat{\mu}, \hat{\sigma}^{2}\right)$, where $\hat{\mu}=\frac{1}{n} \sum_{i=1}^{n} X_{-i}$ and $\hat{\sigma}^{2}=\frac{1}{n-1} \sum_{i=1}^{n}\left(X_{-i}-\hat{\mu}\right)^{2}$. The set of potential parameters is $D_{q}=\mathbb{R} \times(0, \infty)$ which is a subset of the Euclidean space $D=\mathbb{R}^{2}$. The parameters needed to run the chart (3) are just the same as the one needed to update the distribution, thus $\Xi=D_{q}$ and $\xi: D_{q} \rightarrow \Xi,(\mu, \sigma) \mapsto(\mu, \sigma)$ is just the identity.

As before, we are interested in hitting probabilities within the first $T$ steps. Using the function hit defined in the previous subsection, we can write the hitting probability in this parametric setup as hit $^{N}: D_{q} \times \Xi \rightarrow \mathbb{R},(\mu, \sigma ; \xi) \mapsto \operatorname{hit}\left(\Phi_{\mu, \sigma^{2}} ; \xi\right)$, where $\Phi_{\mu, \sigma^{2}}$ is the cdf of the normal distribution with mean $\mu$ and variance $\sigma^{2}$ and the superscript $N$ stands for normal distribution. Furthermore, using $c_{\text {hit }}$ from the previous subsection, the threshold needed to achieve a given hitting probability is $c_{\mathrm{hit}}^{N}: D_{q} \times \Xi \rightarrow \mathbb{R},(\mu, \sigma ; \xi) \mapsto c_{\mathrm{hit}}\left(\Phi_{\mu, \sigma^{2}} ; \xi\right)$.

The resampling is a parametric resampling. To put this in the framework of the main theorem, we let $W_{n}=\left(W_{n 1}, \ldots, W_{n n}\right)$, where $W_{n 1}, \ldots, W_{n n} \sim N(0,1)$ are independent. The resampled parameters are then $\hat{\mu}_{n}^{*}=\frac{1}{n} \sum_{i=1}^{n} X_{n i}^{*}$ and $\hat{\sigma}_{n}^{* 2}=\frac{1}{n-1} \sum_{i=1}^{n}\left(X_{n i}^{*}-\hat{\mu}_{n}^{*}\right)^{2}$ where $X_{n i}^{*}=\hat{P}_{2} W_{n i}+\hat{P}_{1}$.

The following lemma shows that condition a) of Theorem 1 is satisfied.

Lemma 2. For every $\theta \in \mathbb{R} \times(0, \infty)$ and every $\xi \in \mathbb{R} \times(0, \infty)$, the functions hit $^{N}$ and $c_{\text {hit }}^{N}$ are Hadamard differentiable at $\theta$ around $\xi$.

The proof can be found in Appendix B.4, using again the preparatory results of Appendix B.1 - B.3.

Concerning the other conditions of Theorem 1: Condition b) can be shown using standard asymptotic theory, e.g. maximum likelihood theory, which will yield that $Z$ is normally distributed. Condition c) is essentially the requirement that the parametric bootstrap of normally distributed data is working. As $Z$ is a normally distributed vector, condition d) holds unless $q^{\prime}$ equals 0 . Condition e) is satisfied, as the mapping $W_{n} \mapsto \hat{P}^{*}\left(\hat{P}, W_{n}\right)=\left(\hat{\mu}_{n}^{*}, \hat{\sigma}_{n}^{* 2}\right)$ is continuous and hence measurable.

\subsubsection{Setup for Shewhart charts}

For Shewhart charts, the same setup as in the previous two sections can be used, the only difference is the choice of $q$. Conditions b), c) and e) are as in the previous two sections. We conjecture that it is possible to show the Hadamard differentiability more directly, as the properties are available in closed form, see Section 2.2.1.

\section{Simulations for homogeneous observations}

We now illustrate our approach by some simulations using CUSUM charts. The simulations were done in $\mathrm{R}$ (R Development Core Team, 2010).

We use two past sample sizes, $n=50$ and $n=500$. The in-control distribution of $X_{t}$ is $N(0,1)$ and we use 1000 replications and $B=1000$ bootstrap replications. We employ both the parametric bootstrap and the nonparametric bootstrap mentioned in the previous sections. For the parametric bootstrap we used the sample mean and sample standard deviation of $X_{-n}, \ldots, X_{-1}$ as estimates for the mean and the standard deviation of the observations.

For the performance measures ARL, $\log (\mathrm{ARL})$, hit and $\operatorname{logit}($ hit) we use a threshold of $c=3$. For $c_{\mathrm{ARL}}$ we calibrate to an ARL of 100 in control and for $c_{\text {hit }}$ we calibrate to a false alarm probability of $5 \%$ in 100 steps.

We use the CUSUM chart (3) with $\Delta=1$ and $\mu$ and $\sigma$ estimated from the past data. To compute properties such as ARL or hitting probabilities, we use a Markov chain approximation (with 75 grid points), similar to the one suggested in Brook and Evans (1972).

\subsection{Coverage probabilities}

Table 1 contains coverage probabilities of nominal 90\% confidence intervals. These are the one-sided lower confidence intervals given by (1), except for $q=\mathrm{ARL}$ and $\log (A R L)$ where the corresponding upper interval is used. 
Table 1: Coverage probabilities of nominal $90 \%$ confidence intervals for CUSUM charts.

\begin{tabular}{|c|c|c|c|c|}
\hline & \multicolumn{2}{|c|}{ Parametric } & \multicolumn{2}{c|}{ Nonparametric } \\
\hline $\mathrm{q}=$ & $\mathrm{n}=50$ & $\mathrm{n}=500$ & $\mathrm{n}=50$ & $\mathrm{n}=500$ \\
\hline $\mathrm{ARL}$ & 1.000 & 0.929 & 0.999 & 0.944 \\
$\log ($ ARL $)$ & 0.928 & 0.899 & 0.902 & 0.915 \\
hit & 0.923 & 0.896 & 0.878 & 0.910 \\
$\operatorname{logit}($ hit $)$ & 0.892 & 0.893 & 0.870 & 0.904 \\
$c_{\text {ARL }}$ & 0.881 & 0.892 & 0.846 & 0.893 \\
$\log \left(c_{\text {ARL }}\right)$ & 0.896 & 0.895 & 0.868 & 0.904 \\
$c_{\text {hit }}$ & 0.878 & 0.890 & 0.843 & 0.891 \\
$\log \left(c_{\text {hit }}\right)$ & 0.897 & 0.893 & 0.856 & 0.901 \\
\hline
\end{tabular}

The standard deviation of the results is roughly 0.01 .

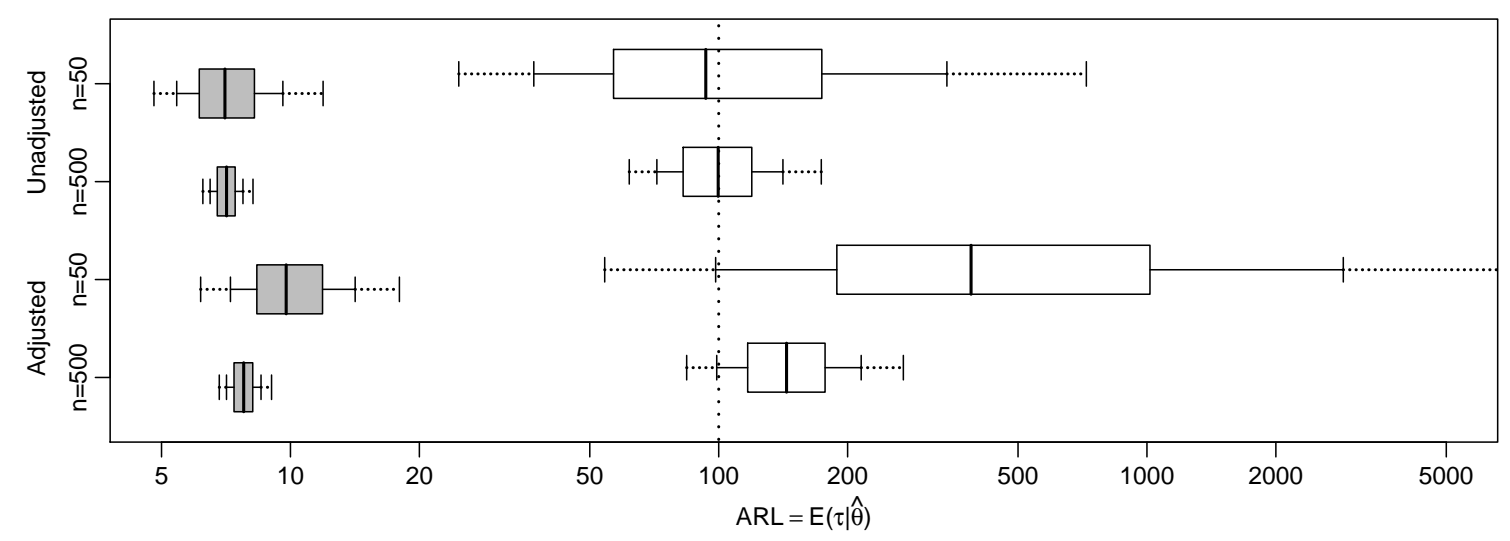

Figure 2: Distribution of the conditional ARL for CUSUMs in a normal distribution setup. Thresholds are calibrated to an in-control ARL of 100. The adjusted thresholds have a guarantee of $90 \%$. A $\log$ transform is used in the calibration. The boxplots show the $2.5 \%, 10 \%, 25 \%, 50 \%, 75 \%, 90 \%$ and $97.5 \%$ quantiles. The white boxplots are in-control, the gray boxplots out-of-control.

In the parametric case, for $n=50$, the coverage probabilities are somewhat off for untransformed versions, in particular for $q=$ ARL. Using log or logit transformations seems to improve the coverage probabilities considerably. In the parametric case, for $n=500$, all coverage probabilities seem to be fine, except for $q=$ ARL, which although shows some marked improvement compare to $n=50$. In the nonparametric case, a similar picture emerges, but the coverage probabilities are a bit worse than in the parametric case.

Remark 3. For $q=\log \left(c_{\mathrm{ARL}}\right)$ and $q=\log \left(c_{\mathrm{hit}}\right)$ the division by $\hat{\sigma}$ in (3) could be skipped without making a difference to the coverage probabilities. Indeed, the division by $\hat{\sigma}$ just scales the chart (and the resulting threshold) by a multiplicative factor, which is turned into an additive factor by $\log$ and which then cancels out in our adjustment.

\subsection{The benefit of an adjusted threshold}

In this section, we consider both the in- and out-of-control performance of CUSUM charts when adjusting the threshold $c$ to give a guaranteed in-control ARL of 100. Setting the threshold is, in our opinion, the most important practical application of our method.

Figure 2 shows average run lengths for both the unadjusted threshold $c(\hat{P} ; \hat{\mu}, \hat{\sigma})$ and the adjusted threshold $\exp \left(\log (c(\hat{P} ; \hat{\mu}, \hat{\sigma}))-p_{0.1}^{*}\right)$, where $p_{0.1}^{*}$ is computed via the parametric bootstrap using $q=\log \left(c_{\mathrm{ARL}}\right)$. Thus, with $90 \%$ probability, the adjusted threshold should lead to an ARL that is 


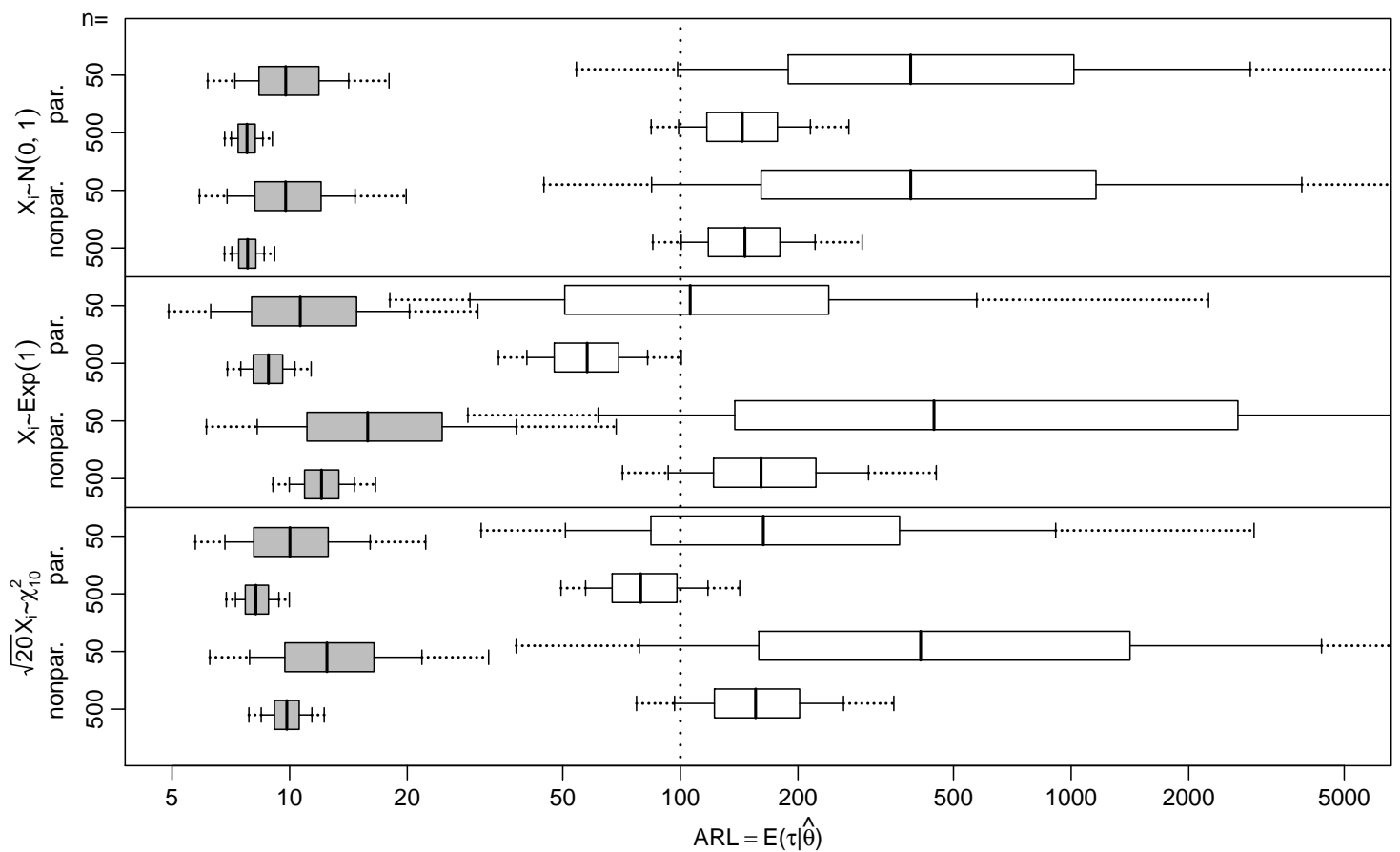

Figure 3: Effects of misspecification. Thresholds are calibrated to an in-control ARL of 100 and adjusted to the estimation error with a guarantee of $90 \%$. A log transform is used in the calibration. The white boxplots are in-control, the gray boxplots are out-of-control. The boxplots show the $2.5 \%, 10 \%, 25 \%, 50 \%, 75 \%, 90 \%$ and $97.5 \%$ quantiles.

above 100. In this and in all following simulations, the out-of-control ARL refers to the situation where the chart is out-of-control from the beginning, i.e. from time 0 onwards.

For the unadjusted threshold, the desired in-control average run length is only reached in roughly half the cases. More importantly, for $n=50$, the probability of having an in-control ARL of below 50 is greater than $20 \%$.

With the adjusted threshold we should get an average run length of at least 100 in $90 \%$ of the cases. This is achieved. The out-of-control ARL using the adjusted thresholds increases only slightly compared to the unadjusted version.

Similarly to Remark 3 , removing the scaling by $\hat{\sigma}$ in (3) would not change the results of this section.

\subsection{Nonparametric bootstrap - advantages and disadvantages}

In this section, we compare the parametric and the non-parametric bootstrap. We consider CUSUM charts that are calibrated to an in-control average run length of 100 assuming a normal distribution. We use the adjusted threshold $\exp \left(\log \left(c_{\mathrm{ARL}}(\hat{P} ; \hat{\mu}, \hat{\sigma})\right)-p_{0.1}^{*}\right)$.

Figure 3 shows the distribution of ARL for $n=50$ and $n=500$ for both the parametric bootstrap that assumes a normal distribution of the updates and the nonparametric bootstrap. We consider both a correctly specified model where $X_{t} \sim N(0,1)$ as well as two misspecified models where $X_{t} \sim \operatorname{Exponential(1)}$ and $\sqrt{20} X_{t} \sim \chi_{10}^{2}$ (all of the $X_{t}$ have variance 1 ). We show both the in- as well as the out-of-control performance of the charts.

In the correctly specified model $\left(X_{t} \sim N(0,1)\right)$, the performance of the parametric and the nonparametric chart seems to be almost identical. The only difference is a slightly worse in-control performance for the non-parametric chart for $n=50$.

In the misspecified model with $X_{t} \sim \operatorname{Exponential(1),~the~parametric~chart~does~not~have~the~}$ desired in-control probabilities. The non-parametric chart seems to be doing well, in particular for $n=500$. We have a similar results in the other misspecified model, with $\sqrt{20} X_{t} \sim \chi_{10}^{2}$. 


\section{Regression models}

In many monitoring situations, the units being monitored are heterogeneous, for instance when monitoring patients at hospitals or bank customers. To make sensible monitoring systems in such situations, the explainable part of the heterogeneity should be accounted for by relevant regression models. The resulting charts are often called risk adjusted, and an overview of some such charts can be found in Grigg and Farewell (2004).

To run risk adjusted charts, the regression model needs to be estimated based on past data, and this estimation needs to be accounted for. Our approach for setting up charts with a guaranteed performance applies also to risk adjusted charts, and we will in particular look at linear, logistic and survival models.

\subsection{Linear models}

Suppose we have independent observations $\left(Y_{1}, X_{1}\right),\left(Y_{2}, X_{2}\right), \ldots$, where $Y_{i}$ is a response of interest and $X_{i}$ is a corresponding vector of covariates, with the first component usually equal to 1 . Let $P$ denote the joint distribution of $\left(Y_{i}, X_{i}\right)$ and suppose that in control $\mathrm{E}\left(Y_{i} \mid X_{i}\right)=X_{i} \xi$. From some observation $\kappa$ there is a shift in the mean response to $\mathrm{E}\left(Y_{i} \mid X_{i}\right)=\Delta+X_{i} \xi$ for $i=\kappa, \kappa+1, \ldots$

Monitoring schemes for detecting changes in regression models can naturally be based on residuals of the model, see for instance Brown et al. (1975) and Horváth et al. (2004). We can, for instance, define a CUSUM to monitor changes in the conditional mean of $Y$ by

$$
S_{t}=\max \left(0, S_{t-1}+Y_{t}-X_{t} \xi-\Delta / 2\right), \quad S_{0}=0,
$$

with hitting time $\tau=\inf \left\{t>0: S_{t} \geq c\right\}$ for some threshold $c>0$. In a similiar manner we could also set up charts for monitoring changes in other components of $\xi$.

The parameter vector $\xi$ is estimated from past in control data, e.g. by the standard least squares estimator. We suggest to use a nonparametric version of the general Algorithm 1 with $\hat{P}$ being the empirical distribution putting weight $1 / n$ on each of the past observations $\left(Y_{-n}, X_{-n}\right), \ldots,\left(Y_{-1}, X_{-1}\right)$. Resampling is then equivalent to resampling $\left(Y_{-n}^{*}, X_{-n}^{*}\right), \ldots,\left(Y_{-1}^{*}, X_{-1}^{*}\right)$ by drawing with replacement from $\hat{P}$.

The suggested method should work even if the linear model is misspecified, i.e. $\mathrm{E}\left(Y_{i} \mid X_{i}\right)=X_{i} \xi$ does not necessarily hold. The nonparametric bootstrap should take this into account.

An analogous approach can be used for Shewhart charts. In settings where it is reasonable to consider the covariate vector to be non-random one could alternatively use bootstrapping of residuals, see for example Freedman (1981).

\subsubsection{Theoretical considerations}

Obtaining precise results is more demanding than in the examples without covariates in Section 3.2 . We only give an idea of the setup that might be used.

The set of distributions of the observations $D_{q}$ can be chosen as the set of cdfs on $\mathbb{R}^{d+1}$ with finite second moments, where $d$ is the dimension of the covariate. The first cdf corresponds to the responses, the others to the covariates. $D_{q}$ is contained in the vector space $D=l_{\infty}\left(\mathbb{R}^{d+1}\right)$, the set of bounded functions $\mathbb{R}^{d+1} \rightarrow \mathbb{R}$. The parameters needed to run the chart are the regression coefficients contained in the set $\Xi=\mathbb{R}^{d}$. These parameters are obtained from the distribution of the observations via $\xi: D_{q} \rightarrow \Xi, F \mapsto\left(E\left(X^{T} X\right)\right)^{-1} E(X Y)$ where $(Y, X) \sim F$ where $X$ is considered to be a row vector.

We conjecture that the conditions of Theorem 1 are broadly satisfied if the cdf of $Y-X \xi$ is differentiable and if for the property $q$ we use hitting probabilities or thresholds to achieve a given hitting probability. In particular, it should be possible to show Hadamard differentiability similarly to Lemma 1. write $q$ as concatenation of two functions and use the chain rule in Lemma 4. The first mapping returns the distribution of the updates of the chart depending on $F \in D_{q}$ and $\xi \in \Xi$ via $(F ; \xi) \mapsto \mathcal{L}(Y-X \xi-\Delta)$, where $\mathcal{L}$ denotes the law of a random variable. The second takes the distribution of the updates and returns the property of interests. The differentiability of the second map has been shown in Lemmas 5 and 6 . 


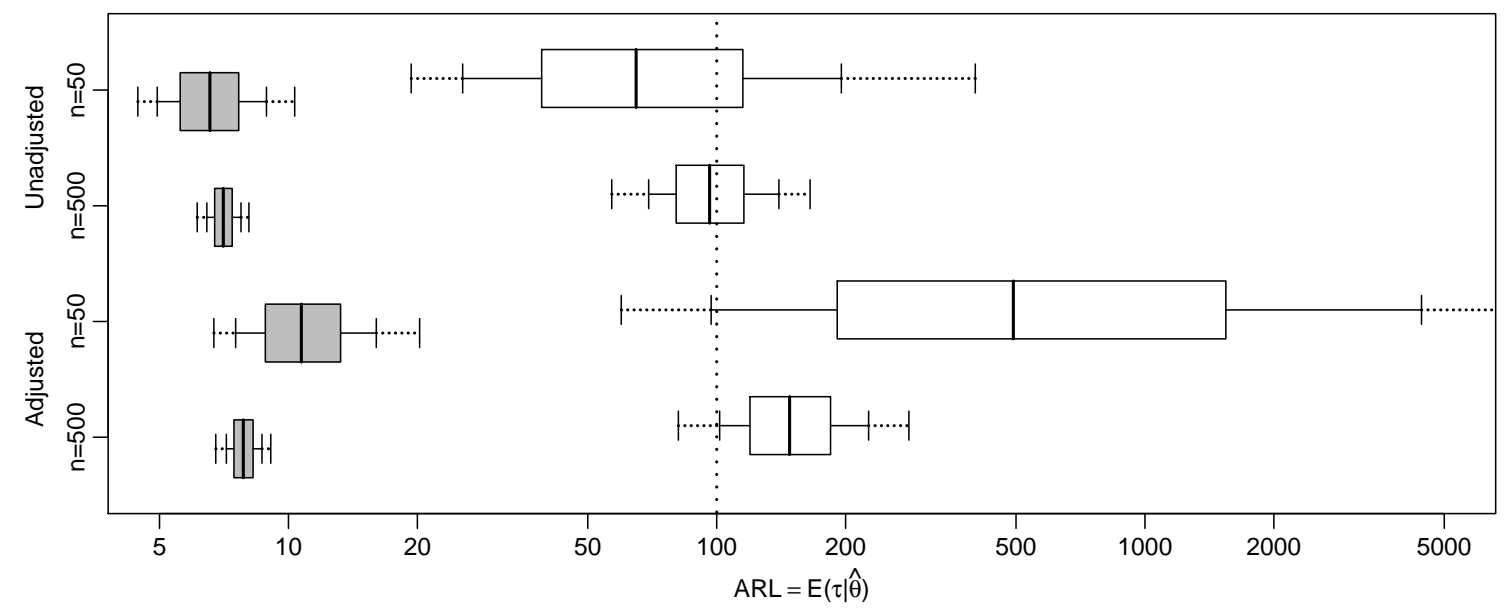

Figure 4: Distribution of the conditional ARL for CUSUMs in a linear regression setup. Thresholds are calibrated to an in-control ARL of 100. A log transform is used in the calibration. The adjusted thresholds have a guarantee of $90 \%$. The white boxplots are in control, the gray out-of-control. The boxplots show the $2.5 \%, 10 \%, 25 \%, 50 \%, 75 \%, 90 \%$ and $97.5 \%$ quantiles.

\subsubsection{Simulations}

We illustrate the performance of the bootstrapping scheme using a CUSUM and the linear in-control model $Y=X_{1}+X_{2}+X_{3}+\epsilon$. Let $\epsilon \sim N(0,1), X_{1} \sim \operatorname{Bernoulli}(0.4), X_{2} \sim U(0,1)$ and $X_{3} \sim N(0,1)$, where $X_{1}, X_{2}, X_{3}$ and $\epsilon$ are all independent. The out-of-control model is $Y=1+X_{1}+X_{2}+X_{3}+\epsilon$, i.e. $\Delta=1$. Figure 4 shows the distribution of the attained ARL for CUSUMs with thresholds calibrated to give an in control ARL of 100. We see that the behaviour of the adjusted versus unadjusted thresholds are very similar to what we observed for the simpler model in Figure 2. The coverage probabilities obtained for this regression model, not reported here, are also very similar to the covarage probabilities reported in Table 1, though with a tendency to be slightly worse.

\subsection{Logistic regression}

Control charts, in particular CUSUM charts, based on logistic regression models are popular for modelling of binary outcomes in medical contexts. See e.g. Lie et al. (1993), Steiner et al. (2000), Grigg and Farewell (2004) and Woodall (2006).

Suppose we have independent observations $\left(Y_{1}, X_{1}\right),\left(Y_{2}, X_{2}\right), \ldots$, where $Y_{i}$ is a binary response variable and $X_{i}$ is a corresponding vector of covariates. Further, suppose that in control the log odds ratio is $\operatorname{logit}\left(\mathrm{P}\left(Y_{i}=1 \mid X_{i}\right)\right)=X_{i} \xi$, and that from some observation $\kappa$ there is a shift in the $\log$ odds ratio to $\operatorname{logit}\left(\mathrm{P}\left(Y_{i}=1 \mid X_{i}\right)\right)=\Delta+X_{i} \xi$ for $i=\kappa, \kappa+1, \ldots$

A CUSUM to monitor changes in the odds ratio can be defined by (Steiner et al., 2000)

$$
S_{t}=\max \left(0, S_{t-1}+R_{t}\right), \quad S_{0}=0,
$$

where $R_{t}$ is the log likelihood ratio between the in-control and out-of-control model for observation $t$. More precisely

$$
\exp \left(R_{t}\right)=\frac{\exp \left(\Delta+X_{t} \xi\right)^{Y_{t}} /\left(1+\exp \left(\Delta+X_{t} \xi\right)\right)}{\exp \left(X_{t} \xi\right)^{Y_{t}} /\left(1+\exp \left(X_{t} \xi\right)\right)}=\exp \left(Y_{t} \Delta\right) \frac{1+\exp \left(X_{t} \xi\right)}{1+\exp \left(\Delta+X_{t} \xi\right)}
$$

The parameter vector $\xi$ is estimated from past in-control data by e.g. the standard maximum likelihood estimator. The same nonparametric bootstrap approach as described for the linear model in Section 5.1 can now be applied to this CUSUM based on this logistic regression model. Moreover, this approach would also apply to control charts based on other generalized linear models, for instance Poisson regression models for monitoring count data. The only amendment needed is to replace $R_{t}$ by the relevant log likelihood ratio. 


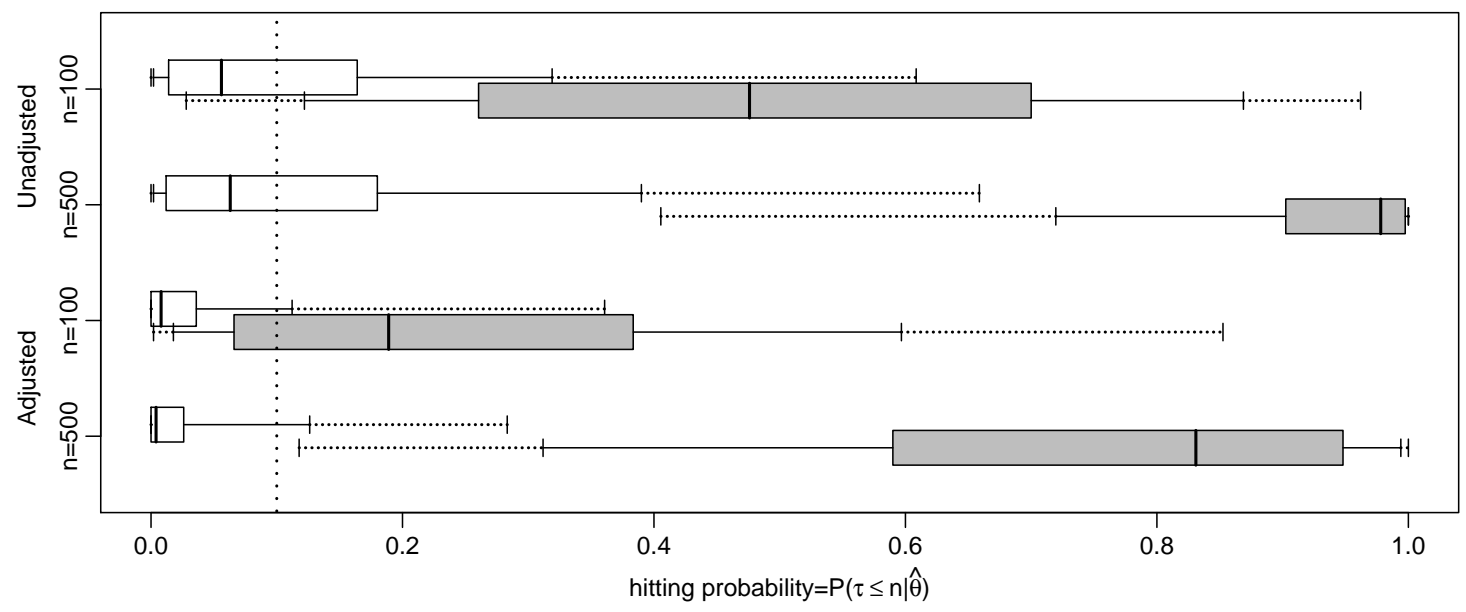

Figure 5: Distribution of the conditional hitting probability for survival analysis CUSUMs. Thresholds are calibrated to an in-control hitting probability of 0.1 . The adjusted thresholds have a guarantee of $90 \%$. The white boxplots are in control, the gray out-of-control. The boxplots show the $2.5 \%, 10 \%, 25 \%, 50 \%, 75 \%, 90 \%$ and $97.5 \%$ quantiles.

We have run simulations, not reported here, based on the same covariate specifications as in Section 5.1.2. The results are similar to the results for the linear model of Section 5.1.2.

\subsection{Survival analysis models}

Recently, risk adjusted control charts based on survival models have started to appear, see Biswas and Kalbfleisch (2008); Sego et al. (2009); Steiner and Jones (2009); Gandy et al. (2010). In none of these papers any adjustment for estimation error is done, but Sego et al. (2009) are illustrating, by simulations, the impact of estimation error on the attained average run length for the accelerated failure time model based CUSUM studied in their paper.

In the following, we provide a brief simulation example of our adjustment in a survival setup where we use the methods described in Gandy et al. (2010).

We observe the survival of individuals over a fixed time interval of length $n$ (we will use $n=100$ and $n=500$ ). Individuals arrive at times $B_{i}$ (in our simulation according to a Poisson process with rate 1 ), and survive for $T_{i}$ time units. Individuals may arrive before the observation interval, as long as $B_{i}+T_{i}$ is after the start of the observation interval. Right-censoring, at $C_{i}$ time units after arrival, is taking place after a maximum follow-up time of $t=60$ time units or after the individuals leave the observation interval. In the simulation, the true hazard rate of $T_{i}$ is $h_{i}(t)=0.1 \exp \left(X_{1 i}+X_{2 i}\right)$, where $X_{1 i} \sim \operatorname{Bernoulli}(0.4)$ and $X_{2 i} \sim N(0,1)$ are covariates.

Based on the observed data we fit a Cox proportional hazard model with $X_{1 i}$ and $X_{2 i}$ as covariates and nonparametric baseline, giving estimates $\hat{\beta}$ for the covariate effects and $\hat{\Lambda}_{0}(t)$ for the the integrated baseline.

We use the CUSUM chart described in Gandy et al. (2010) against a proportional alternative with $\rho=1.25$. The parameters needed to run the chart are $\xi=\left(\beta, \Lambda_{0}\right)$ estimated by $\hat{\xi}=\left(\hat{\beta}, \hat{\Lambda}_{0}\right)$. To be precise, the chart signals at time $\tau=\inf \{t>0: S(t) \geq c\}$, where $S(t)=R(t)-\inf _{s \leq t} R(s)$, $R(t)=\log (\rho) N(t)-(\rho-1) \Lambda(t), N(t)$ is the number of events until time $t$ and $\Lambda(t)=\sum_{i} \exp \left(\beta_{1} X_{i 1}+\right.$ $\left.\beta_{2} X_{2 i}\right) \Lambda_{0}\left(\min \left(\left(t-B_{i}\right)^{+}, T_{i}, C_{i}\right)\right)$.

We are interested in finding a threshold that gives a desired hitting probability, i.e. we use $q=c_{\text {hit }}$. We compute $c_{\text {hit }}(P, \xi)$ via simulations (simulate new data from $P$ and run the chart with $\xi$ ). We estimate the threshold needed to get a $10 \%$ false alarm probability in $n$ time units in control, by the $90 \%$ quantile of 500 simulations of the maximum of the chart.

To resample, we resample individuals with replacement. We use 500 bootstrap samples. Figure 5 shows the distribution of the resulting hitting probabilities based on 500 simulated observation intervals. 
In control, without the adjustment, the desired false alarm probability of 0.1 is only reached in roughly $60 \%$ of the cases. The bootstrap correction seems to work fine, leading to a false alarm probability of at most $10 \%$ in roughly $90 \%$ of the cases. As expected, increasing the length of the fitting period and the length of time the chart is run from $n=100$ to $n=500$ results in higher out-of-control hitting probabilities.

If the length of the fitting period and the deployment period of the chart differ then a somewhat more complicated resampling procedure needs to be used. For example, one could resample arrival times and survival times/covariates separately. The former could be done by assuming a Poisson process as arrival time and the latter either by resampling with replacement or by sampling from an estimated Cox model and an estimated censoring distribution.

\section{Conclusions and discussion}

We have presented a general approach for handling estimation error in control charts with estimated parameters and unknown in-control distributions. Our suggestion is, by bootstrap methods, to tune the monitoring scheme to guarantee, with high probability, a certain conditional in-control performance (conditional on the estimated in-control distribution). If we apply a nonparametric bootstrap, the approach is robust against model specification error.

In our opinion, focusing on a guaranteed conditional in-control performance is generally more relevant than focusing on some average performance, as an estimated chart usually is run for some time without independent reestimation. Our approach can also easily be adapted to make for instance bias adjustments. Bias adjustments, in contrast to guaranteed performance, tend to be substantially influenced by tail behaviour for heavy tailed distributions which for instance the average run length has. This implies that the bias adjustments need not be useful in the majority of cases as the main effect of the adjustment is to adjust the tail behaviour.

We have in particular demonstrated our approach for various variants of Shewhart and CUSUM charts, but the general approach will apply to other charts as well. The method is generally relevant when the in-control distribution is unknown and the conditions of Theorem 11 hold. We conjecture that this will be the case for many of the most commonly used control charts. Numerous extensions of control charts to other settings exist, for example to other regression models, to autocorrelated data, to multivariate data. We do conjecture that our approach will also apply in many of these settings.

\section{A Proof of the main theorem}

The following extension of the functional delta method will help in the proof of Theorem 1

Lemma 3. Suppose that $q: D_{q} \times \Xi \rightarrow E$ is Hadamard differentiable at $P \in D_{q}$ around $\xi \in \Xi$ tangentially to $D_{0} \subset D$ and that $\xi: D_{q} \rightarrow \Xi$ is continuous. Let $\hat{P}$ be a sequence of random elements in $D_{q}$ such that

$$
\sqrt{n}(\hat{P}-P) \rightsquigarrow Z \quad(n \rightarrow \infty),
$$

where $Z$ is some tight random element in $D_{0}$. Then

$$
\sqrt{n}(q(\hat{P} ; \xi(\hat{P}))-q(P ; \xi(\hat{P}))) \rightsquigarrow q^{\prime}(P ; \xi(P)) Z .
$$

Proof. Note that $\sqrt{n}(q(\hat{P} ; \hat{\xi})-q(P ; \hat{\xi}))=g_{n}(\sqrt{n}(\hat{P}-P))$, where $g_{n}: \tilde{D}_{n} \rightarrow F, g_{n}(h)=\sqrt{n}[q(P+$ $\left.\left.n^{-\frac{1}{2}} h ; \xi\left(P+n^{-\frac{1}{2}} h\right)\right)-q\left(P ; \xi\left(P+n^{-\frac{1}{2}} h\right)\right)\right]$ and $\tilde{D}_{n}=\left\{h \in D: P+n^{-\frac{1}{2}} h \in D_{q}\right\}$.

Let $h_{n}$ be a sequence such that $h_{n} \in \tilde{D}_{n}$ and $h_{n} \rightarrow h$ for some $h \in D_{0}$. Let $\xi_{n}=\xi\left(P+n^{-\frac{1}{2}} h_{n}\right)$. The continuity of $\xi$ implies $\xi_{n} \rightarrow \xi(P)$. Thus by the Hadamard differentiability of $q$ we get $g_{n}\left(h_{n}\right) \rightarrow$ $q^{\prime}(P ; \xi(P))(h)$. Using the extended continuous mapping theorem (van der Vaart and Wellner, 1996, Th 1.11.1) finishes the proof.

Proof of Theorem 1. Let $\tilde{Z}_{1}$ and $\tilde{Z}_{2}$ be independent copies of $Z$. Arguing as in the first part of the proof of (Kosorok, 2008, Theorem 12.1) one can see that unconditionally

$$
\sqrt{n}\left(\left(\begin{array}{c}
\hat{P}^{*} \\
\hat{P}
\end{array}\right)-\left(\begin{array}{c}
P \\
P
\end{array}\right)\right) \rightsquigarrow\left(\begin{array}{c}
\tilde{Z}_{1}+\tilde{Z}_{2} \\
\tilde{Z}_{2}
\end{array}\right) .
$$


Applying Lemma 3 to this with the mappings $(x, y ; \xi) \mapsto(q(x ; \xi), q(y ; \xi), x, y)$ and $(x, y) \mapsto \xi(x)$ gives

$$
\sqrt{n}\left(\begin{array}{c}
q\left(\hat{P}^{*} ; \hat{\xi}^{*}\right)-q\left(P ; \hat{\xi}^{*}\right) \\
q\left(\hat{P} ; \hat{\xi}^{*}\right)-q\left(P ; \hat{\xi}^{*}\right) \\
\hat{P}^{*}-P \\
\hat{P}-P
\end{array}\right) \rightsquigarrow\left(\begin{array}{c}
q^{\prime}(P ; \xi)\left(\tilde{Z}_{1}+\tilde{Z}_{2}\right) \\
q^{\prime}(P ; \xi)\left(\tilde{Z}_{2}\right) \\
\tilde{Z}_{1}+\tilde{Z}_{2} \\
\tilde{Z}_{2}
\end{array}\right) .
$$

After that one can argue exactly as in the remainder of the proof of (Kosorok, 2008, Theorem 12.1), p.237, to show that

$$
A_{n}:=\sqrt{n}\left(q\left(\hat{P}^{*} ; \xi\left(\hat{P}^{*}\right)\right)-q\left(\hat{P} ; \xi\left(\hat{P}^{*}\right)\right) \underset{W}{\stackrel{P}{\leftrightarrow}} G \quad(n \rightarrow \infty)\right.
$$

for $G=q^{\prime}(P ; \xi) Z$. Furthermore, Lemma 3 shows that

$$
B_{n}:=\sqrt{n}(q(\hat{P} ; \hat{\xi})-q(P ; \hat{\xi})) \rightsquigarrow G .
$$

Similarly to the ideas in (van der Vaart, 1998, Lemma 23.3), one can show that (5) and (6) imply the correct coverage probabilities. Indeed, for any subsequence there is a further subsequence such that $A_{n} \rightsquigarrow G$ a.s. conditionally on $\hat{P}$. Using (van der Vaart, 1998, Lemma 21.2), we get $F_{A_{n}}^{-1} \rightsquigarrow F_{G}^{-1}$ along this subsequence, where $F^{-1}$ denotes the quantile function of the random variable in the subscript, i.e. $F_{G}^{-1}(x)=$ $\inf \{t \in \mathbb{R}: P(G \leq t) \geq x\}$. Thus for any continuity point $\beta$ of $F_{G}^{-1}$, we get $F_{A_{n}}^{-1}(\beta) \rightarrow F_{G}^{-1}(\beta)$ a.s. along the subsequence. Thus overall, we have

$$
F_{A_{n}}^{-1}(\beta) \stackrel{P}{\rightarrow} F_{G}^{-1}(\beta) .
$$

By Slutsky's lemma and $\left[6, B_{n}-F_{A_{n}}^{-1}(\beta) \rightsquigarrow G-F_{G}^{-1}(\beta)\right.$. Thus, as $G$ is continuous,

$$
\mathrm{P}\left(B_{n} \leq F_{A_{n}}^{-1}(\beta)\right) \rightarrow \mathrm{P}\left(G \leq F_{G}^{-1}(\beta)\right)=\beta .
$$

This holds for all $\beta$, because there are at most countably many points $\beta$ at which $F_{G}^{-1}$ is not continuous, because both the left- and the right-hand side of $(7)$ are monotone in $\beta$, and because the right-hand side is continuous. As $p_{\alpha}^{*}=F_{A_{n}}^{-1}(\alpha) / \sqrt{n}, \sqrt{7}$ implies

$$
\mathrm{P}\left(q(P ; \hat{\xi})<q(\hat{P} ; \hat{\xi})-p_{\alpha}^{*}\right)=1-\mathrm{P}\left(q(\hat{P} ; \hat{\xi})-q(P ; \hat{\xi}) \leq p_{\alpha}^{*}\right)=1-\mathrm{P}\left(B_{n} \leq F_{A_{n}}^{-1}(\alpha)\right) \rightarrow 1-\alpha .
$$

\section{B Proofs for Hadamard differentiability}

The main goal of this section is to prove Lemmas 1 and 2 . Before we do this in Appendix B.4. we first show a chain rule in Appendix B.1 then a lemma about the uniform Hadamard differentiability of inverse maps in Appendix B.2. After that we show general differentiability of hitting probability in CUSUM charts with respect to the updating distribution in Appendix B.3 The results in B.1. B.3 may also be useful in other situations.

\section{B.1 Chain rule}

In this section we present a chain rule for Hadamard differentiable functions. For this we need the following stronger version of Hadamard differentiability.

Definition 2. Let $D, E$ be metric spaces. A function $\phi: D_{\phi} \subset D \rightarrow E$ is called uniformly Hadamard differentiable at $\theta \in D_{\theta}$ along $d: D \times D \rightarrow \mathbb{R}$ tangentially to $D_{0} \subset D$ if there exists a linear map $\phi_{\theta}^{\prime}: D_{0} \rightarrow E$ such that

$$
\frac{\phi\left(\theta_{n}+t_{n} h_{n}\right)-\phi\left(\theta_{n}\right)}{t_{n}} \rightarrow \phi_{\theta}^{\prime}(h)
$$

for all $\theta_{n} \rightarrow \theta$ with $d\left(\theta_{n}, \theta\right) \rightarrow 0, t_{n} \rightarrow 0$ and all converging sequences $\left(h_{n}\right)$ with $h_{n} \rightarrow h \in D_{0}$ and $\theta_{n}+t_{n} h_{n} \in D_{\phi}$.

Lemma 4 (Chain rule). Let $D, E, F$ be metric spaces and let $H$ be a non-empty set. Let $\left\{f_{\xi}: D_{f} \rightarrow\right.$ $E: \xi \in \Xi\}$ be a family of functions that is Hadamard differentiable at $\theta \in D_{f}$ around $\xi \in \Xi$ tangentially to $D_{0} \subset D$. Let $\phi: E_{\phi} \rightarrow F$ be uniformly Hadamard differentiable at $f_{\xi}(\theta)$ along $d: E_{\phi} \times E_{\phi} \rightarrow \mathbb{R}$ tangentially to $f_{\xi}^{\prime}(\theta ; \xi)\left(D_{0}\right)$. Furthermore, suppose that $\xi_{n} \rightarrow \xi$ implies $d\left(f\left(\theta ; \xi_{n}\right), f(\theta ; \xi)\right) \rightarrow 0$. Then $\left\{\phi \circ f_{\xi}: D_{f} \rightarrow F: \xi \in \Xi\right\}$ is Hadamard differentiable at $\theta$ around $\xi \in \Xi$ tangentially to $D_{0}$. 
Proof. Let $\left(\xi_{n}\right) \subset \Xi,\left(t_{n}\right) \subset \mathbb{R},\left(h_{n}\right) \subset D$ satisfying $\theta+t_{n} h_{n} \in D_{f} \forall n$ and $\xi_{n} \rightarrow \xi, t_{n} \rightarrow 0, h_{n} \rightarrow h \in D_{0}$ as $n \rightarrow \infty$. Let $k_{n}=\frac{f_{\xi_{n}}\left(\theta+t_{n} h_{n}\right)-f_{\xi_{n}}(\theta)}{t_{n}}$. Hadamard differentiability of $f$ implies $k_{n} \rightarrow q_{\xi}^{\prime}(h)$. Then by uniform Hadamard differentiability of $\phi$,

$$
\frac{\phi\left(f_{\xi_{n}}\left(\theta+t_{n} h_{n}\right)\right)-\phi\left(f_{\xi_{n}}(\theta)\right)}{t_{n}}=\frac{\phi\left(f_{\xi_{n}}(\theta)+t_{n} k_{n}\right)-\phi\left(f_{\xi_{n}}(\theta)\right)}{t_{n}} \rightarrow \phi_{f_{\xi}(\theta)}^{\prime}\left(q_{\xi}^{\prime}(\theta)(h)\right) .
$$

\section{B.2 Uniform Hadamard differentiability of the inverse map}

Let $D_{\phi}$ be the set of non-decreasing functions in $D[u, v]$, for some $-\infty<u<v<\infty$, that cross $\beta \in \mathbb{R}$, i.e.

$$
D_{\phi}=\{F \in D[u, v]: F \text { non-decreasing, } \exists x \in(u, v]: F(x-) \leq \beta \leq F(x)\} .
$$

Suppose that $F \in D_{\phi}$ and $G \in D_{\phi}$ are differentiable on $[u, v]$ with derivatives $f$ and $g$. Let $d(F, G)=$ $\sup _{x \in[u, v]}|f(u)-g(u)|$. If either $F$ or $G$ are not differentiable on $[u, v]$ then we set $d(F, G)=\infty$.

Let $\phi: D_{\phi} \rightarrow \mathbb{R}, \phi(F)=\inf \{x: F(x) \geq \beta\}$, the first point at which the function crosses the threshold.

Lemma 5. Let $\theta \in D_{\phi}$ such that $\theta$ is differentiable on $[u, v]$ with continuous bounded positive derivative. Then $\phi$ is uniformly Hadamard differentiable at $\theta$ along $d$ tangentially to $C[u, v]$.

Proof. Let $\xi=\phi(\theta)$. Let $\left(h_{n}\right) \subset D[u, v]$ such that $h_{n} \rightarrow h \in C[u, v]$. Let $\left(t_{n}\right) \subset[0, \infty)$ such that $t_{n} \rightarrow 0$. Let $\left(\theta_{n}\right) \subset D_{\phi}$ such that $\theta_{n} \rightarrow \theta$ and $d\left(\theta_{n}, \theta\right) \rightarrow 0$. Let $\xi_{n}=\phi\left(\theta_{n}+t_{n} h_{n}\right)$. By the definition of $\phi$, we have

$$
\left(\theta_{n}+t_{n} h_{n}\right)\left(\xi_{n}-\epsilon_{n}\right) \leq \beta \leq\left(\theta_{n}+t_{n} h_{n}\right)\left(\xi_{n}\right) .
$$

for every $\epsilon_{n}>0$. Let $\left(\epsilon_{n}\right)$ be positive and such that $\epsilon_{n}=o\left(t_{n}\right)$.

First, we show $\xi_{n} \rightarrow \xi$. The sequence $\left(h_{n}\right)$ is uniformly bounded because $h_{n} \rightarrow h$ and because $h$ is bounded. Thus,

As $t_{n} \rightarrow 0$ and $\theta_{n} \rightarrow \theta$

$$
\theta_{n}\left(\xi_{n}-\epsilon_{n}\right)+O\left(t_{n}\right) \leq \beta \leq \theta_{n}\left(\xi_{n}\right)+O\left(t_{n}\right) .
$$

$$
\theta\left(\xi_{n}-\epsilon_{n}\right)+o(1) \leq \beta \leq \theta\left(\xi_{n}\right)+o(1)
$$

For every $\delta>0$, the function $\theta$ is bounded away from $\beta$ outside $(\xi-\delta, \xi+\delta)$. Furthermore, $\theta$ is strictly increasing. Thus, to satisfy the previous display we must have eventually $\xi_{n} \geq \xi-\delta$ and $\xi_{n}-\epsilon_{n} \leq \xi+\delta$, which implies $\xi_{n} \rightarrow \xi$.

Let $\tilde{\xi}_{n}=\phi\left(\theta_{n}\right)$. Using the mean value theorem in (8) gives

$$
\theta_{n}\left(\tilde{\xi}_{n}\right)+\left(\xi_{n}-\epsilon_{n}-\tilde{\xi}_{n}\right) \theta_{n}^{\prime}\left(\rho_{1 n}\right)+t_{n} h_{n}\left(\xi_{n}-\epsilon_{n}\right) \leq \beta \leq \theta_{n}\left(\tilde{\xi}_{n}\right)+\left(\xi_{n}-\tilde{\xi}_{n}\right) \theta_{n}^{\prime}\left(\rho_{2 n}\right)+t_{n} h_{n}\left(\xi_{n}\right)
$$

for some $\rho_{1 n}$ between $\xi_{n}-\epsilon_{n}$ and $\tilde{\xi}_{n}$ and for some $\rho_{2 n}$ between $\xi_{n}$ and $\tilde{\xi}_{n}$. . Rewriting this using $\theta_{n}\left(\tilde{\xi}_{n}\right)=\beta$ gives

$$
\left(\xi_{n}-\tilde{\xi}_{n}\right) \theta_{n}^{\prime}\left(\rho_{1 n}\right)+t_{n} h_{n}\left(\xi_{n}-\epsilon_{n}\right)-\epsilon_{n} \theta_{n}^{\prime}\left(\rho_{1 n}\right) \leq 0 \leq\left(\xi_{n}-\tilde{\xi}_{n}\right) \theta_{n}^{\prime}\left(\rho_{2 n}\right)+t_{n} h_{n}\left(\xi_{n}\right) .
$$

By the uniform convergence of $h_{n}$ and the continuity of $h$, we have $h_{n}\left(\xi_{n}-\epsilon_{n}\right) \rightarrow h(\xi)$ and $h_{n}\left(\xi_{n}\right) \rightarrow h(\xi)$. Using this, the fact that we have chosen $\epsilon_{n}$ such that $\epsilon_{n}=o\left(t_{n}\right)$ and that $\theta_{n}^{\prime}$ is uniformly bounded, we get

$$
\left(\xi_{n}-\tilde{\xi}_{n}\right) \theta_{n}^{\prime}\left(\rho_{1 n}\right)-o\left(t_{n}\right) \leq-t_{n} h(\xi) \leq\left(\xi_{n}-\tilde{\xi}_{n}\right) \theta_{n}^{\prime}\left(\rho_{2 n}\right)+o\left(t_{n}\right)
$$

Hence,

$$
-\frac{h(\xi)}{\theta_{n}^{\prime}\left(\rho_{2 n}\right)}-o(1) \leq \frac{\xi_{n}-\tilde{\xi}_{n}}{t_{n}} \leq-\frac{h(\xi)}{\theta_{n}^{\prime}\left(\rho_{1 n}\right)}+o(1) .
$$

We have already shown $\xi_{n} \rightarrow \xi$ which implies $\rho_{1 n} \rightarrow \xi$ and $\rho_{2 n} \rightarrow \xi$. Using the assumptions that $\theta_{n}^{\prime} \rightarrow \theta$ uniformly and that $\theta^{\prime}$ is continuous shows $\theta_{n}^{\prime}\left(\rho_{1 n}\right) \rightarrow \theta^{\prime}(\xi)$ and $\theta_{n}^{\prime}\left(\rho_{2 n}\right) \rightarrow \theta^{\prime}(\xi)$, which finishes the proof. 


\section{B.3 Differentiability of the hitting probability with respect to the up- dating distribution}

We are interested in hitting probabilities for CUSUM charts within the first $T \in \mathbb{N}$ steps. We will show that the mapping from the distribution of the updates $Y_{i}$ to the hitting probabilities is uniformly Hadamard differentiable. The $Y_{i}$ are the adjusted observations, e.g. in the notation of Section 3.2.1 they are $Y_{i}=\frac{X_{i}-\xi_{1}-\Delta / 2}{\xi_{2}}$, where $X_{i}$ is the observed value.

Let $D_{\phi}^{\xi_{2}}$ be the set of cumulative distribution functions on $\mathbb{R}$, considered as a subset of $D=l_{\infty}(\mathbb{R})$ equipped with the uniform norm. Consider the mapping

$$
\phi: D_{\phi} \rightarrow l_{\infty}(\mathbb{R}), q(F)(c)=P(\text { hit threshold c within } \mathrm{T})=\int g(y, c) d F\left(y_{1}\right) \ldots d F\left(y_{T}\right),
$$

where $g(y, c)=1(m(y) \geq c)$ with $1(\cdot)$ being the indicator function and $m(y)$ is as defined in Section 3.2.1.

Lemma 6. $\phi$ is uniformly Hadamard differentiable tangentially to $D_{0}=\left\{H \in C(\mathbb{R}): \lim _{t \rightarrow \infty} H(t)=\right.$ $\left.\lim _{t \rightarrow-\infty} H(t)=0\right\}$ with derivative

$$
\phi^{\prime}(F)(H)(c)=\sum_{i=1}^{T} \int g(y, c)\left(\prod_{j \neq i} d F\left(y_{j}\right)\right) d H\left(y_{i}\right)
$$

Since $H$ may be of infinite variation, the above integral is defined by partial integration, i.e.

$$
\phi^{\prime}(F)(H)(c)=-\sum_{i=1}^{T} \int H\left(y_{i}\right) d\left(\int g(y, c)\left(\prod_{j \neq i} d F\left(y_{j}\right)\right)\right) .
$$

Proof. Suppose $F_{n} \rightarrow F, t_{n} \rightarrow 0, H_{n} \rightarrow H \in D_{0}$ such that $F_{n}+t_{n} H_{n} \in D_{\phi}$ for all $n$. The difference quotient can be written as

$$
\frac{\phi\left(F_{n}+t_{n} H_{n}\right)(c)-\phi\left(F_{n}\right)(c)}{t_{n}}=\sum_{i=1}^{T} \int g(y, c)\left(\prod_{j \neq i} d F_{n}\left(y_{j}\right)\right) d H_{n}\left(y_{i}\right)+\sum_{\substack{I \subset\{1, \ldots, T\} \\|I| \geq 2}} t_{n}^{|I|-1} A_{I},
$$

where $A_{I}=\int g(y, c)\left(\prod_{i \notin I} d F_{n}\left(y_{i}\right)\right)\left(\prod_{i \in I} d H_{n}\left(y_{i}\right)\right)$. We first show that the second terms converges uniformly in $c$ to 0 . Partial integration (applied several times) gives that for $I \subset\{1, \ldots, T\},|I| \geq 2$,

$$
A_{I}=(-1)^{|I|} \int\left(\prod_{i \in I} H_{n}\left(y_{i}\right)\right) d B_{I}\left(y_{I}\right)
$$

where $B_{I}=\int g(y, c) \prod_{i \notin I} d F_{n}\left(y_{i}\right) . \quad g(y, c)$ is monotonically increasing in $y$ thus $B_{I}$ is increasing in $y_{I}=\left(y_{i}\right)_{i \in I}$. Thus the total variation of $B_{I}$ is bounded by 1 . Hence, using (10),

$$
t_{n}^{|I|-1} A_{I} \leq t_{n}^{|I|-1}\left(\sup _{z \in \mathbb{R}}\left|H_{n}(z)\right|\right)^{|I|}
$$

which converges to 0 uniformly in $c$. Thus the second term of 9 converges to 0 uniformly in $c$.

Next, we show that first term on the right hand side of (9), henceforth denoted by $\zeta$, converges uniformly in $c$ to $\phi^{\prime}(F)(H)$. Consider the decomposition

$$
\zeta-\phi^{\prime}(F)(H)=C_{n}+\sum_{i=1}^{T} \int g(y, c)\left(\left(\prod_{j \neq i} d F_{n}\left(y_{j}\right)\right)-\prod_{j \neq i} d F\left(y_{j}\right)\right) d H\left(y_{i}\right),
$$

where $C_{n}=\sum_{i=1}^{T} \int g(y, c)\left(\prod_{j \neq i} d F_{n}\left(y_{j}\right)\right)\left(d H_{n}\left(y_{i}\right)-d H\left(y_{i}\right)\right)$. As mentioned above, $H$ might be of infinite variation, thus $C_{n}$ is defined via partial integration, i.e.

$$
C_{n}=-\sum_{i=1}^{T} \int\left(H_{n}\left(y_{i}\right)-H\left(y_{i}\right)\right) d\left(\int g(y, c) \prod_{j \neq i} d F_{n}\left(y_{j}\right)\right) .
$$

As above, the total variation of the integrator is bounded by 1 , thus $\left|C_{n}\right| \leq T\left\|H_{n}-H\right\|$, which converges to 0 as $n \rightarrow \infty$. 
We can rewrite the second term of 11$]$ as

$$
\sum_{i=1}^{T} \sum_{k=1, k \neq i}^{T} \int D_{i k}\left(\left(\prod_{j \neq i, j<k} d F_{n}\left(y_{j}\right)\right) \prod_{j \neq i, j>k} d F\left(y_{j}\right)\right) .
$$

where $D_{i k}=\int g(y, c) d H\left(y_{i}\right)\left(d F_{n}\left(y_{k}\right)-d F\left(y_{k}\right)\right)$. Using partial integration,

$$
D_{i k}=-\int H\left(y_{i}\right) d g\left(y_{-i}, d y_{i}, c\right)\left(d F_{n}\left(y_{k}\right)-d F\left(y_{k}\right)\right)=\int H\left(y_{i}\right)\left(F_{n}\left(y_{k}\right)-F\left(y_{k}\right)\right) d g\left(y_{-i,-k}, d y_{i}, d y_{k}, c\right),
$$

where negative subscripts denote removal of the corresponding component of the vector (e.g. $y_{-i}$ is the vector $y$ with the $i$ th component removed). Since $g$ is of bounded variation with respect to $y_{i}$ and $y_{k}$ independent of $c$ and $y_{-i,-k}$, we can bound this uniformly above by $K \sup _{z}|H(z)| \sup _{z}\left|F_{n}(z)-F(z)\right|$ for some fixed $K>0$. Thus, since the variation of $F_{n}-F$ is bounded by 2 , the second term of (11) converges to 0 uniformly in $c$.

The following lemma is needed to use the result about the inverse mapping, see Lemma 5.

Lemma 7. Let $F$ be a cumulative distribution function with continuous bounded positive derivative $f$. Let $Y=\left(Y_{1}, \ldots, Y_{T}\right)$ where $Y_{1}, \ldots, Y_{T} \sim F$ independently. Then the following holds.

a) $c \mapsto \mathrm{P}(m(Y) \leq c)$ is continuously differentiable for $c>0$ (call this derivative $g$ ).

b) $g$ is bounded away from 0 (at least on some compact sets),

c) Let $f_{n}$ be densities that converge uniformly to $f$. Let $Y^{(n)}=\left(Y_{1}^{(n)}, \ldots, Y_{T}^{(n)}\right) \sim f_{n}$ and denote the derivative of $c \mapsto \mathrm{P}\left(m\left(Y^{(n)}\right) \leq c\right)$ for $c>0$ by $g^{(n)}$. Then $g^{(n)}$ converges uniformly to $g$ on any compact set $K \subset(0, \infty)$.

Proof. For $0 \leq i \leq T$, let $A_{i}=\left\{y \in \mathbb{R}^{T}: R_{i}(y)>R_{\nu}(y) \forall \nu \neq i\right\}$. $A_{i}$ are disjoint sets with $\mathrm{P}\left(Y \in \bigcup_{i} A_{i}\right)=$ 1. Thus, $\mathrm{P}(m(Y) \leq c)=\sum_{i} \mathrm{P}\left(m(Y) \leq c, Y \in A_{i}\right)$ and $g(c)=\sum_{i=1}^{T} g_{i}(c)$, where

$$
\begin{aligned}
& g_{i}(c)=\frac{\partial}{\partial c} \mathrm{P}\left(m(Y) \leq c, Y \in A_{i}\right)=\frac{\partial}{\partial c} \mathrm{P}\left(R_{i}(Y) \leq c, Y \in A_{i}\right) \\
& =\frac{\partial}{\partial c} \mathrm{P}\left(Y_{i} \leq c-R_{i-1}(Y), Y \in A_{i}\right)=\int \frac{\partial}{\partial c} \int^{c-R_{i-1}(y)} 1\left(y \in A_{i}\right) f\left(y_{i}\right) d y_{i} \prod_{\nu \neq i} f\left(y_{\nu}\right) d y_{-i} \\
& =\int 1\left(\left(y_{1}, \ldots, y_{i-1}, c-R_{i-1}(y), y_{i+1}, \ldots, y_{T}\right) \in A_{i}\right) f\left(c-R_{i-1}(y)\right) \prod_{\nu \neq i} f\left(y_{\nu}\right) d y_{-i} .
\end{aligned}
$$

The continuity of $g$ follows because because of the dominated convergence theorem. This shows a). Statement b) follows from $g$ being positive and its continuity. For c), use a telescoping sum to go from the product of $f \mathrm{~s}$ to the product of $f_{n} \mathrm{~s}$. Then use the dominated convergence theorem.

\section{B.4 Hadamard differentiability of hitting probability in simple examples}

Lemma 1, hit can be written as $\phi \circ g$, where $\phi$ is as in Appendix B.3 and $g: D_{q} \rightarrow D_{q}, g(P ; \xi)=(x \mapsto$ $P\left(x \xi_{2}+\xi_{1}+\Delta / 2\right)$.

We will show that $g$ is Hadamard differentiable at $P$ around $\xi$ tangentially to $D_{0}$. Clearly, $g$ is linear in $P$. Thus for $t_{n} \rightarrow 0, h_{n} \rightarrow h \in D_{0}, \xi_{n} \rightarrow \xi$,

$$
\begin{aligned}
\frac{g\left(P+t_{n} h_{n} ; \xi_{n}\right)-g\left(P ; \xi_{n}\right)}{t_{n}}-g(h ; \xi) & =g\left(h_{n} ; \xi_{n}\right)-g(h ; \xi) \\
& =\left(g\left(h_{n} ; \xi_{n}\right)-g\left(h ; \xi_{n}\right)\right)+\left(g\left(h ; \xi_{n}\right)-g(h ; \xi)\right) .
\end{aligned}
$$

The first term converges uniformly to 0 as $h_{n} \rightarrow h$. The second term converges to 0 as $h \in D_{0}$ implies that $h$ is uniformly continuous.

Lemma 6 allows us to use the chain rule in Lemma 4, to show the differentiability of hit.

The differentiability of $c_{\text {hit }}$ can be seen as follows: $\xi_{n} \rightarrow \xi$ implies that the derivative of $g\left(P ; \xi_{n}\right)$ converges uniformly to the derivative of $g(P ; \xi)$. As $g\left(P ; \xi_{n}\right)^{\prime}(x)=f\left(x \xi_{2 n}+\xi_{1 n}+\Delta / 2\right) \xi_{2 n}$ this is implied by the uniform continuity of $f$. Thus, by Lemma 7 , the derivative of hit $\left(P ; \xi_{n}\right)$ converges uniformly to the derivative of $\operatorname{hit}(P ; \xi)$. Thus, the result follows using the chain rule (Lemma 4), the differentiability of hit, and the differentiability of the inverse (Lemma 5).

Lemma 2. Let $g:(\mathbb{R} \times(0, \infty))^{2} \rightarrow D_{q}, g(\mu, \sigma, \xi)=\left(x \mapsto \Phi\left(\frac{\xi_{1}+\Delta / 2+\xi_{2} x-\mu}{\sigma}\right)\right)$. Then, as in the proof of Lemma 1. hit $^{N}=\phi \circ g$. The proof can be completed with similar steps. 


\section{References}

Albers, W. and W. C. Kallenberg (2004a). Are estimated control charts in control? Statistics 38, 67-79.

Albers, W. and W. C. Kallenberg (2004b). Estimation in Shewart control charts: effects and corrections. Metrika 59, 207-234.

Albers, W. and W. C. Kallenberg (2005). New corrections for old control charts. Quality Engineering 17, 467-473.

Albers, W. and W. C. Kallenberg (2006). Self-adapting control charts. Statistica Neerlandica 60, $292-308$.

Albers, W. and W. C. Kallenberg (2009). Cumin charts. Metrika 70, 111-113.

Albers, W. and W. C. Kallenberg (2010). The optimal choice of negative binomial charts for monitoring high-quality processes. Journal of Statistical Planning and Inference 140, 214-225.

Albers, W., W. C. Kallenberg, and S. Nurdiati (2005). Exceedance probabilities for parametric control charts. Statistics 39, 429-443.

Aue, A., L. Horváth, M. Hušková, and P. Kokoszka (2006). Change-point monitoring in linear models. Econometrics Journal 9, 373-403.

Biswas, P. and J. D. Kalbfleisch (2008). A risk-adjusted CUSUM in continuous time based on the Cox model. Statist. Med. 27(17), 3382-3406.

Bottle, A. and P. Aylin (2008, Feb). Intelligent information: a national system for monitoring clinical performance. Health Serv Res 43(1 Pt 1), 10-31.

Brook, D. and D. A. Evans (1972). An approach to the probability distribution of cusum run length. Biometrika 59(3), 539-549.

Brown, R., J. Durbin, and J. Evans (1975). Techniques for testing the constancy of regression relationships over time (with discussion). J. R. Statist. Soc. B 37, 149-192.

Capizzi, G. and G. Masarotto (2009). Bootstrap-based design of residual control charts. IIE Transactions 41(4), $275-286$.

Carey, R. G. (2003). Improving Healthcare with Control Charts: Basic and Advanced SPC Methods and Case Studies. ASQ Quality Press, Milwaukee.

Castagliola, P. and P. E. Maravelakis (2011). A cusum control chart for monitoring the variance when parameters are estimated. Journal of Statistical Planning and Inference 141, 1463-1478.

Chakraborti, S. and M. Graham (2007). Nonparametric control charts. In F. Ruggeri, R. Kenett, and F. Faltin (Eds.), Encyclopedia of Statistics in Quality and Reliability, pp. 415-429. Wiley.

Champ, C. W. and L. A. Jones-Farmer (2007). Properties of multivariate control charts with estimated parameters. Sequential Analysis: Design Methods and Applications 26(2), 153-169.

Chatterjee, S. and P. Qiu (2009). Distribution-free cumulative sum control charts using bootstrap-based control limits. Ann. Appl. Stat. 3, 349-369.

Fouladirad, M., A. Grall, and L. Dieulle (2008). On the use of on-line detection for maintenance of gradually deteriorating systems. Reliability Engineering and System Safety 93, 1814-1820.

Freedman, D. A. (1981). Bootstrapping regression models. Ann. Statist. 9(6), 1218-1228.

Frisén, M. (Ed.) (2008). Financial Surveillance. Wiley.

Frisén, M. and E. Andersson (2009). Semiparametric surveillance of monotonic changes. Sequential Analysis 28(4), 434-454.

Gandy, A., J. Kvaløy, A. Bottle, and F. Zhou (2010, June). Risk-adjusted monitoring of time to event. Biometrika 97(2), 375-388.

Grigg, O. and V. Farewell (2004). An overview of risk-adjusted charts. J. R. Statist. Soc. A. 167(3), 523-539.

Hawkins, D. M. (1987). Self-starting cusum charts for location and scale. J. R. Statist. Soc. D 36(4), 299-316.

Hawkins, D. M. and D. H. Olwell (1998). Cumulative Sum Charts and Charting for Quality Improvement. Springer.

Horváth, L., M. Hušková, P. Kokoszka, and J. Steinebach (2004). Monitoring changes in linear models. Journal of Statistical Planning and Inference 126, 225-251.

Hušková, M. and C. Kirch (2010). Bootstrapping sequential change-point tests for linear regression. Preprint.

Jensen, W. A., L. A. Jones-Farmer, C. W. Champ, and W. H. Woodall (2006). Effects of parameter estimation on control chart properties; a literature review. Journal of Quality Technology Vol. 38(4), 349-364.

Jones, L. A. (2002). The statistical design of EWMA control charts with estimated parameters. Journal of Quality Technology 34(3), 277-288.

Jones, L. A., C. W. Champ, and S. E. Rigdon (2004). The run length distribution of the cusum with estimated parameters. Journal of Quality Technology 36(1), 95-108. 
Kirch, C. (2008). Bootstrapping sequential change-point tests. Sequential Analysis 27, 330-349.

Kosorok, M. R. (2008). Introduction to Empirical Processes and Semiparametric Inference. Springer.

Lawson, A. B. and K. Ken (Eds.) (2005). Spatial and syndromic surveillance for public health. Wiley.

Lie, R., I. Heuch, and L. Irgens (1993). A new sequential procedure for surveillance of Down's syndrome. Statist. Med. 12, 13-25.

Maravelakis, P. E. and P. Castagliola (2009). An ewma chart for monitoring the process standard deviation when parameters are estimated. Computational Statistics and Data Analysis 53, 2653-2664.

Moustakides, G. V. (1986). Optimal stopping times for detecting changes in distributions. Ann. Statist. 14(4), 1379-1387.

O'Connor, P. D. (2002). Practical reliability engineering. Wiley.

Page, E. S. (1954). Continuous inspection schemes. Biometrika 41(1/2), 100-115.

R Development Core Team (2010). R: A language and environment for statistical computing. R Foundation for Statistical Computing.

Sego, L. H., M. R. Reynolds, Jr, and W. H. Woodall (2009). Risk-adjusted monitoring of survival times. Statist. Med. 28(9), 1386-1401.

Shewhart, W. A. (1931). Economic Control of Quality of Manufactured Product. Van Nostrand, New York.

Steiner, S. H., R. J. Cook, V. T. Farewell, and T. Treasure (2000). Monitoring surgical performance using risk-adjusted cumulative sum charts. Biostat 1(4), 441-452.

Steiner, S. H. and M. Jones (2009). Risk-adjusted survival time monitoring with an updating exponentially weighted moving average (ewma) control chart. Statis. Med. 29, 444-454.

Stoumbos, Z. G., J. Marion R. Reynolds, T. P. Ryan, and W. H. Woodall (2000). The state of statistical process control as we proceed into the 21st century. J. Am. Statist. Ass. 95(451), 992-998.

Sullivan, J. H. and L. A. Jones (2002). A self-starting control chart for multivariate individual observations. Technometrics $44(1), 24-33$.

van der Vaart, A. (1998). Asymptotic Statistics. Cambridge University Press.

van der Vaart, A. and J. Wellner (1996). Weak Convergence and Empirical Processes. Springer.

Woodall, W. H. (2006). The use of control charts in health-care and public-health surveillance. Journal of Quality Technology 38(2), 89-134. With discussion.

Xie, M., T. Goh, and P. Ranjan (2002). Some effectice control chart procedures for reliability monitoring. Reliability Engineering and System Safety 77, 143-150.

Zhang, Y., P. Castagliola, Z. Wu, and M. B. C. Khoo (2011). The synthetic $\bar{X}$ chart with estimated parameters. IIE Transactions 43, 676-687. 